\title{
Demokratische Republik Kongo - die Verfassung von 2005 (I)
}

\author{
Von Dietrich Nelle, Brüssel
}

\section{Einleitung}

Im nach Fläche und Bevölkerung dritt- bzw. viertgrößten Land Afrikas herrscht seit der Verkündung der neuen Verfassung im Februar 2006 Aufbruchstimmung. Staatspräsident Kabila hat die Übergangszeit für beendet erklärt und die III. Republik ausgerufen. Noch versuchen allerdings Milizen diese Wahlen zu verhindern und amtierende Politiker zumindest einen Aufschub ihrer Abwahl zu erreichen. Der von den Bürgerkriegsereignissen besonders betroffene, ehemals nach afrikanischen Maßstäben wohlhabende Osten des Landes ist immer noch nicht zur Ruhe gekommen. Rebellen üben in weiten Gebieten die Staatsgewalt aus und treiben Phantomsteuern von einer verängstigten Bevölkerung ein. Insgesamt ist der Kongo eines der 11 am wenigsten entwickelten Länder der Erde ${ }^{1}$. Die weitere Entwicklung dieses an der Schnittstelle von arabischen und schwarzafrikanischen, von franko-, luso- und anglophon geprägten Staaten gelegenen Landes verspricht schicksalhaft für den gesamten Kontinent zu werden. Stabilisierung und Schaffung neuen Wohlstands sind entscheidende Voraussetzungen für ein Nachlassen des Auswanderungsdrucks nach Europa, Nordamerika und in andere afrikanische Staaten. Vor diesem Hintergrund hat die Europäische Union, welche ohnehin der größte Geber im Land ist, eine führende Rolle im Stabilisierungsprozess übernommen. Die Verfassung von 2005/2006 als ein wesentlicher Baustein in diesem Prozess wird im folgenden Beitrag in ihren wesentlichen Inhalten erläutert und in den aktuellen Entwicklungskontext des Landes gestellt.

\section{Allgemeine Geschichte/Verfassungsgeschichte}

a) Verfassungsdokumente der Kolonialzeit

Die Verfassungsgeschichte des Landes ist durch eine rasche Abfolge zahlreicher Verfassungen gekennzeichnet. Eine erste Rezeption westlichen Verfassungsrechts erfolgte bereits zu Beginn des 16. Jh., als der kongolesische König Alfonso zum Christentum übertrat und das portugiesische Regierungsmodell übernahm. Im 19. Jahrhundert lenkten diverse Reise- 
berichte - u.a. von David Livingstone, der die östlichen Regionen untersuchte, und Pierre Savorgnan de Brazza, der von Gabun ins Kongobecken vordrang - das Interesse auf diese Region als geheimnisvolle Quelle unerhörten Reichtums. Großen Einfluss hatte der angloamerikanische Journalist Sir Henry Morton Stanley, der 1877 den Kongo befuhr und weit ins Landesinnere vordrang. Angeregt von Stanley errichtete der belgische König Leopold II. im Jahr 1879 eine Handelsgesellschaft, die Stanley beauftragte, in das Gebiet zurückzukehren, um Handelsstationen $\mathrm{zu}$ errichten und freundschaftliche Beziehungen $\mathrm{zu}$ den Stammeshäuptlingen aufzubauen. Er gründete u.a. die Stadt Léopoldville (das heutige Kinshasa) und sicherte durch Protektoratsverträge die Rechte an weiten Gebieten entlang des Kongo. In der Berliner Konferenz 1884 wurde das Gebiet gegen konkurrierende Ansprüche Portugals und Frankreichs der belgischen Interessenssphäre als „Kongo-Freistaat" zugeschlagen und weniger später dem belgischen König in Personalunion unterstellt $^{2}$. Nachdem das Bekanntwerden erheblicher Missstände zu Diskussionen in ganz Europa führte, übernahm der belgische Staat die Verantwortung, indem er das Gebiet 1908 in eine Kolonie überführte. Grundlegendes Dokument der belgischen Kolonialzeit war die Kolonialcharta desselben Jahres ${ }^{3}$, welche vor allem auf eine Trennung zwischen eingewanderten Weißen und assimilierten Einheimischen auf der einen Seite und den übrigen, in ihrer Lebensweise weniger berührten Einheimischen auf der anderen Seite setzte. Nach dem Ersten Weltkrieg wurde das Gebiet zeitweilig mit den benachbarten ehemals deutschen Kolonien Burundi und Ruanda verbunden.

\section{b) Verfassungsdokumente der Übergangszeit 1960-67}

\section{aa) Verfassung des Brüsseler Runden Tisches von 1960}

Bei Eintritt in die Unabhängigkeit war der Kongo ein wirtschaftlich vergleichsweise wohlhabendes Land auf einem Niveau ähnlich wie Südafrika oder Kanada ${ }^{4}$. Der Übergang zur Unabhängigkeit des Kongo wurde aber spät und unzureichend vorbereitet. Erst 1957 konnte die autochthone Bevölkerung erstmals an den Wahlen zu den Verwaltungsbezirken teilnehmen. Da die erste Hochschule des Landes erst 1954 gegründet wurde, hatte sich auch kaum eine intellektuelle Elite heranbilden können. Nachdem jedoch General de

G.L. Ulmen, Dialectic of Darkness, reflections on the Congo Conference and the Collapse of the Ius Publicum, in: Dietrich Murswiek (Hrsg.), Staat-Souveränität-Verfassung, FS für Helmut Quaritsch, Berlin 2000, S 693 ff.

3 Loi belge v 18.10.1908 über die Regierung des belgischen Kongo, BO 1908, S 65 ff.; vgl. John Crabb, The Legal System of Congo-Kinshasa, Charlottesville, Va. 1970, 43 ff.; Giovanni Buccianti, Il Congo, Mailand 1963, S 305 ff.

4

Modeste Mutinga, Chronique d'une paix négocié en Rdc, Kinshasa, 2005, 9. 
Gaulle 1959 in einer berühmt gewordenen Rede, welche er in Brazzaville, also in Sichtweite der kongolesischen Hauptstadt Léopoldville hielt, den bisherigen französischen Besitzungen die Unabhängigkeit in Aussicht gestellt hatte, wurden im auch im Kongo die Forderungen nach umgehender Eigenständigkeit immer lauter ${ }^{5}$. Als es 1959 zu Unruhen kam, versuchte das Mutterland den völligen Verlust seiner wirtschaftlich bedeutenden Kolonie zunächst durch das Angebot erhöhter Autonomie abzuwenden; es zeigte sich jedoch rasch, dass die umgehende Gewährung staatlicher Unabhängigkeit für den Kongo unvermeidbar war ${ }^{6}$. Am 30. Juni 1960 proklamierte der belgische König in Léopoldville die Demokratische Republik Kongo.

Kurz zuvor war in aller Eile ein Runder Tisch einberufen worden, um ein Übergangsgrundgesetz ${ }^{7}$ für den neuen Staat vorzubereiten. Allerdings litten die Arbeiten nicht nur unter dem enormen Zeitdruck, sondern mehr noch an den mangelnden Gestaltungsmöglichkeiten der noch völlig unorganisierten künftigen politischen Elite des neuen Landes ${ }^{8}$. Die wirklichen Entscheidungen wurden vielmehr im belgischen Kolonialministerium als Sekretariat des Runden Tisches vorbereitet und von den beiden Kammern des belgischen Parlaments verabschiedet und durch den König verkündet ${ }^{9}$. So überrascht es nicht, dass das neue Grundgesetz weitgehend den belgischen Parlamentarismus spiegelte und bei den neuen Akteuren wenig innere Akzeptanz fand ${ }^{10}$. Das Verfassungsdokument erkannte dies auch selber an, indem es seine baldige Ablösung durch eine von den Kongolesen selber ausgearbeitete und den örtlichen Mentalitäten angepassten Verfassung ankündigte (Art. 3). Zu den offensichtlichsten Mängeln gehörte, dass angesichts der im Referenzland geltenden Staatsform der Monarchie kein Verfahren für eine Neubestellung des Staatsoberhauptes geregelt wurde. Auch konnte die komplexe föderale Struktur an keine einheimische Traditionen anknüpfen. Als besonders verhängnisvoll erwiesen sich die unklaren Regelungen zur Aufgabenteilung zwischen Staatsoberhaupt und Regierungschef, welche die unmittelbar nach Unabhängigkeit bereits aufflammenden internen Machtkämpfe nach der Unabhängigkeit

Kambayi Bwatshia, La Faiblesse des parties politiques congolais à l'origine du chaos au Congo, Le Phare (Kinshasa) v. 21.10.2004.

6 Kambayi Bwatshia, (Fn. 5).

7

Loi belge v. 19.5.1960 über die Strukturen des Kongo, MC 1960 I 1535 ff.; engl Übersetzung bei Peaslee, Constitutions of Nations, 3. Aufl. Den Haag 1965, S. 98 ff.; Giovanni Buccianti (Fn. 3), S. 333 ff.; vgl ferner Ajami, Die Verfassung der Demokratischen Republik Kongo, Köln 1970, S 4 ff.; Wilfried Rather, Die Verfassungsentwicklung und Verfassungswirklichkeit Zaires (19601989), JÖR 38 (1989), S. 526 ff.; J-L Kasambo Kangashe, Evolution constitutionnelle et perspectives électorales en RDC, Le Potentiel (Kinshasa) v. 3.3.2005.

Kambayi Bwatshia (Fn. 5).

9 Esambo Kangashe Evolution constitutionnelle et perspectives électorales en RDC, Le Potentiel (Kinshasa) v. 3.3.2005.

10 Vgl Ajami (Fn. 7). 
noch anheizten bis hin zur wechselseitigen Amtsenthebung von Staatspräsident und Premierminister, zu ethnischen Konflikten, zu gewalttätigen Auseinandersetzungen und zur Sezessionserklärung der rohstoffreichen Katanga-Provinz. Erst nach erneuter militärischer Intervention der ehemaligen Kolonialmacht trat wieder eine gewisse Beruhigung ein. Die belgischen Streitkräfte wurden in der Folge durch VN-Streitkräfte abgelöst, welche sich allerdings zuvor verpflichten mussten, nicht in interne Konflikte einzugreifen. Der Militäroberbefehlshaber und spätere langjährige Diktator Mobutu nutzte die chaotischen Zustände, um eine geschäftsführende Regierung unter seiner Kontrolle zu etablieren, doch gelang es dem Staatspräsidenten nach einem halben Jahr wieder die Oberhand gewinnen und selber eine provisorische Regierung einzusetzen.

Wenig später versah der Sicherheitsrat die VN-Truppen mit einem "robusten" Mandat, ohne damit allerdings die Ordnung wiederherstellen zu können. Die Sezessionsbestrebungen in Katanga gingen weiter; der mit Vermittlungsbemühungen befasste seinerzeitige VNGeneralsekretär Dag Hammarskjöld ${ }^{11}$ kam unter bis heute ungeklärten Umständen bei einem Flugzeugabsturz im heutigen Sambia ums Leben. Eine Gruppe von Führern politischer Gruppierungen versuchte sogar, den Zentralstaat durch einen Bund souveräner Staaten zu ersetzen. 1963 schlugen die VN-Truppen schließlich die Sezession von Katanga gewaltsam nieder.

\section{bb) Luluabourg-Verfassung von 1964}

Nach diesem Einschnitt wurde eine Regierung der nationalen Einheit gebildet. Auch diese konnte allerdings keine dauerhafte Beruhigung erreichen. Nachdem der frühere Sezessionsführer die Leitung der Zentralregierung übernommen hatte, sah dieser sich seinerseits einer Rebellion ausgesetzt, die er nur mit Hilfe belgischer Truppen und weißer Söldner niederzuschlagen vermochte.

Vor diesem Hintergrund erfolgte unter Betreuung der $\mathrm{VN}^{12}$ die Ausarbeitung der ersten eigenen Verfassung des Landes. Hierzu fanden eine Vielzahl politischer Konferenzen und Konklaven statt, welche schließlich zu einem Konsens führten, welcher in vielen Punkten Kompromisscharakter trug und oft eine klare Positionierung vermied ${ }^{13} . \mathrm{Zu}$ den Kennzei-

Durch die Verordnung Nr 298 v 24.11.1963 wurde eine ausschließlich aus Kongolesen bestehende Kommission von 112 Mitgliedern eingesetzt, welche vor allem vom amtierenden VN-Generalsekretär U Thant und dem nigerianischen Juristen Alawele beraten wurde, vgl. Esambo Kangashe (Fn.9).

Ajami (Fn. 7)., Die Verfassung der Demokratischen Republik Kongo, Köln 1970, S 8 ff.; J-L Kasambo Kangashe (Fn. 7). 
chen dieses Textes gehörten die Verbindung einer präsidialen Ausrichtung mit einer starken Stellung des Parlaments sowie einem Premierminister mit hervorgehobener Rolle sowie die Einführung eines Bundesstaates, in welchem die Provinzen nicht nur über eigenständige Kompetenzen verfügten, sondern großen Einfluss auf die zentralstaatliche Gesetzgebung und auch bei der indirekten Wahl des Staatsoberhaupt ausüben sollten. Diese sog Luluabourg-Verfassung ${ }^{14}$ gilt dennoch vielen Kongolesen noch heute als die einzige authentische kongolesische Verfassung. Sie trat 1964 nach einem erfolgreichen Referendum in Kraft. Auf ihrer Grundlage konnten im Folgejahren noch Parlamentswahlen durchgeführt werden, die inneren Spannungen konnten aber auch auf Grundlage dieses Rechtsdokuments nicht gelöst werden.

Nach massiven internen Machtkämpfen übernahm schließlich im November 1965 Generalleutnant Mobutu die Macht und billigte sich in seiner ersten Gesetzesverordnung selber das Recht zur Gesetzgebung zu ${ }^{15}$. Das zunächst noch vorgesehene Erfordernis der parlamentarischen Ratifikation solcher Verordnungen wurde wenige Monate später gestrichen ${ }^{16}$. Außerdem wurden zunächst für eine Dauer von 5 Jahren alle politischen Parteien verboten und schließlich wichtige Funktionen der Rechtspflege dem neuen Präsidenten zugewie$\operatorname{sen}^{17}$. Weitere fundamentale Abweichungen von Grundentscheidungen der LuluabourgVerfassung bildeten ebenfalls 1966 die Abschaffung des Amtes des Premierministers sowie die Umwandlung der föderativen Struktur in ein zentralistisches Gebilde mit nur 8 statt der vorgesehenen 21 Provinzen ${ }^{18}$. Auch auf wirtschaftlichem Gebiet wurde die Abkehr von marktwirtschaftlichen Grundsätzen eingeleitet und zunächst wichtige ausländische Bergbaubetriebe verstaatlicht.

Die europäischen Namen einiger Städte wurden durch afrikanische Namen ersetzt (so wurde Léopoldville zu Kinshasa und Stanleyville zu Kisangani).

Kongolesische Verfassung v1.8.1964, MC Sonderausgabe v 1.8.1964, S 1 ff.; vgl Ajami, (Fn. 7), S. 8 ff.; Wilfried Rather, (Fn. 7), S. 530 ff.; Tshilombo Munyengayi, A partir du 24 novembre 1965, Mobutu poignarde et viole la Constitution de Luluabourg, Le Potentiel (Kinshasa) v 29.11.2005.

Tshilombo Munyengayi, (Fn.14).

17

Tshilombo Munyengayi, (Fn.14).

18

Tshilombo Munyengayi, (Fn.14). 
Nachdem so die Luluabourg-Verfassung Schritt für Schritt materiell weithin geradezu in ihr Gegenteil verkehrt worden war, ging Mobutu daran, den neuen Verfassungszustand auch offiziell in einem neuen Grundgesetz festzuschreiben, der sog. N'Sele-Verfassung ${ }^{19}$. Ziel sollte die Anpassung der Verfassung an die afrikanischen Realitäten sein ${ }^{20}$. Mobutu verkündete als Eckpunkte u.a. die Abschaffung des politischen Pluralismus, die - erstmalig seit der Unabhängigkeit - Direktwahl des Staatspräsidenten, die Einführung des Frauenwahlrechts, die Absenkung des aktiven und passiven Wahlrechts auf 19 bzw 25 Jahre sowie die Einführung eines Ein-Kammer-Parlaments ${ }^{21}$. Die demgemäß ausgearbeitete Verfassung $^{22}$ wurde in einer Volksabstimmung mit einer offiziellen Zustimmung von 97,8\% gebilligt. Neben den bereits erwähnten Punkten wurde der Staatspräsident auch Regierungschef (Art 20, 29, 30) und erhielt das Recht, jede Verfassungsänderung oder jedes andere Gesetz unmittelbar dem Volk per Referendum vorzulegen (Art 74). Ferner erfolgte die Umwandlung von einem Bundes- in einen Zentralstaat (Art 1). Noch am Tag der Verkündung löste Mobutu das 1965 gewählte Parlament auf ${ }^{23}$ und beseitigte damit das letzte Relikt der Luluabourg-Verfassung. Ferner wurde die Revolutionäre Volksbewegung MPR als neue Einheitspartei gegründet, alle ethnischen und separatistischen Organisationen aufgelöst sowie die zentrale Struktur des Staates mit nunmehr neun Provinzen festgeschrieben. Auch wenn zu keinem Zeitpunkt die völlige Liquidation der Zivilgesellschaft gelang ${ }^{24}$ und es auch immer wieder zu Protesten und Aufständen kam, hatte Mobutu damit seine politische Macht vorläufig gefestigt. Gleichzeitig wurde der Prozess der wirtschaftlichen Umgestaltung fortgesetzt und 1973 in großem Umfang Unternehmen verstaatlicht. Da zudem die für den Kongo besonders wichtigen Kupferpreise stark gefallen waren, verschlechterte sich die wirtschaftliche Lage: Arbeitslosigkeit, Auslandsverschuldung und Inflation stiegen rapide, nationale und internationale Investitionen blieben aus.

21 Tshilombo Munyengayi (Fn. 14). Originaltext und deutscher Übersetzung; J-L Kasambo Kangashe, (Fn.7). Tshilombo Munyengayi (Fn. 14).

24 Besonders wichtige Elemente der Zivilgesellschaft waren die katholischen und protestantischen Kirchen, die Musik sowie die Literatur, vgl Langana et al.(Fn. 51),: un bilan globalement négatif, Le Phare (Kinshasa) v. 15.2.2005, S 2 ff.; Alphonse Mbuyamba Kankolopngo, Littérature congolaise et démocratisiation en Afrique contemporaine, Le Potentiel (Kinshasa) v 4.3.2006. 
Von den durch die N'Sele-Verfassung gegebenen Möglichkeiten zur weiteren Veränderung der Verfassung machte Mobutu in den folgenden Jahren immer wieder Gebrauch. Durch die Verfassungsänderung von $1970^{25}$ wurde die MPR zur einzigen legalen politischen Partei (Art 67). 1971 wurden der Staatsname und das Staatsemblem geändert ${ }^{26}$. Die 1974 vorgenommene Verfassungsänderung ${ }^{27}$ machte die bereits vollzogene Abkehr von demokratischen Grundsätzen geradezu idealtypisch sichtbar. Die Staatsgewalt wurde danach vom Volk nicht mehr wie bisher über Wahlen und Referenden ausgeübt (Art 2 der N'Sele-Verfassung), sondern es wurde in Anlehnung an sozialistische Vorbilder bestimmt, dass dies durch den Vorsitzenden der Einheitspartei geschehen sollte, welcher von Amts wegen auch das Amt des Staatspräsidenten versah (Art $9 \mathrm{nF})^{28}$. Der Staatspräsident saß überdies dem Politbüro, dem Kongress, dem Gesetzgebungsrat und dem Obersten Richterrat vor (Art $30)^{29}$. Als offizielle Staatsdoktrin wurde der "Mobutismus" festgeschrieben (Art 33).

1977/78 kam es in der Katanga-Provinz (1972-1997 Shaba genannt) zu erneuten Kämpfen, als Aufständische von Angola aus eindrangen, um Mobutu zu stürzen. Diese Angriffe wurden mit Hilfe von Belgien, Frankreich und Marokko niedergeschlagen. Dadurch geschwächt musste Mobutu in der Verfassung von $1978^{30}$ erste Einschränkungen seiner absoluten Macht hinnehmen ${ }^{31}$. 1980 erfolgten weitere Korrekturen; insbesondere wurde im Februar die Regelung der Wahl des in seiner Bedeutung abgewerteten Politbüros gestrichen und dieses im Dezember durch ein Zentralkomitee ersetzt ${ }^{32}$. Ein weiterer Autoritätsverlust des Machthabers zeigte sich 1982 in der Schaffung einer Regelung über die Vertretung des

Gesetz Nr 70/001 v 23.12.1970; vgl Wilfried Rather (Fn.7), 38 (1989), S. 531 ff.

Gesetze Nr 71/006 v 29.10.1971 bzw 71/007 v 19.11.1971.

Gesetz v 15.8.1974; vgl Wilfried Rather (Fn. 7), S. 532 ff.

Angelo Mobateli, Afin que nul n'en ignore le contenu avant le référendum, nécessité de vulgariser le projet de Constitution, le Potentiel (Kinshasa) v 28.5.2005; Esambo Kangashe (Fn. 9).

29 Esambo Kangashe (Fn. 9).

30 Vgl. Vanderlinden, Länderbericht Zaire in Blaustein/Flanz (Hrsg.), Constitutions of the Countries of the World, New York 1978 zugleich mit französischer Originalfassung und englischer Übersetzung.

31 Wilfried Rather, (Fn. 7), S. 534 ff.; Durieux, Réforme de la Constitution du 15 février 1978 du Zaïre, RevJurPol 1980, S. 709 ff.; Lions, La constitución de 15 de febrero de 1978 de la Republica de Zaire, Boletín mexicano de derecho comparado 35 (1979), S 519 ff.

Gesetz Nr 80-12 v 15.8.1978; vgl. Wilfried Rather (Fn. 7), S. 534 ff.; Durieux (Fn. 31), S. 859 ff.; Lions, (Fn. 31), S. $519 \mathrm{ff}$. 
Präsidenten im Verhinderungsfall ${ }^{33} .1988$ schließlich wurde die Bestellung der Mitglieder des Zentralkomitee neu geregelt ${ }^{34}$.

d) Verfassungsdokumente der zweiten Phase der II. Republik 1990-2003

aa) Verfassungsentwicklung 1990-1994

Bis zum Ende der 80er-Jahre des vergangenen Jahrhunderts hatten der zunehmende wirtschaftliche und gesellschaftliche Zerfall im Inneren die Stellung des Alleinherrschers Mobutu stark geschwächt. Als äußere Faktoren kamen die anhaltende Instabilität in Nachbarländern wie Burundi und Ruanda sowie der seit 1989 schwindende Ost-West-Gegensatz $^{35}$ hinzu. So sah sich Mobutu zu ersten Reformmaßnahmen gezwungen. Nach Streiks und schweren Unruhen musste Mobutu 1990 auch Oppositionsparteien wieder zulassen und den bisherigen Oppositionsführer als neuen Ministerpräsidenten akzeptieren.

Allerdings genügten diese Schritte nicht, um dem weiteren Verfall Einhalt zu gebieten. Eine 1991 einberufene Nationalkonferenz über die politische Zukunft Zaires ${ }^{36}$ erarbeitete zwar im Laufe eines Dreivierteljahres einen Verfassungsentwurf ${ }^{37}$. Der Oberste Gerichtshof erklärte daraufhin auch die bisherige N'Sele-Verfassung für aufgehoben ${ }^{38}$. Gleichwohl weigerte sich der versierte Machtpolitiker Mobutu, sie durch Verkündung in Kraft zu setzen $^{39}$. Vielmehr berief er seinerseits ein Konklave ein, welches ebenfalls einen neuen Verfassungsentwurf ${ }^{40}$ ausarbeitete. Überdies löste er die Versammlung auf, nachdem sie einen „Hohen Rat der Republik“ als Übergangsparlament gewählt hatte, in welchem die Opposition die Mehrheit besaß. Die konkurrierende Regierungen mit oppositioneller bzw. dem Staatspräsidenten loyaler Ausrichtung bestanden aber fort, ohne das eine der beiden Seiten wirkliche Handlungsfähigkeit besaß.

Gesetze v 9.2.1980 bzw 31.12.1982, verö JO Nr 1 v 1.1.1983, vgl Wilfried Rather (Fn. 7) S. 534 ff.

34

35

Theodor Trefon, The Social Cost of Conflict in the Democratic Republic of the Congo: Crisis, Population, Environment, Oslo 2002.

36

37

38

39

40

Rechtsakt Nr 001/CNS/92; vgl. Esambo Kangashe, (Fn. 9).

Urteil R.A. 266 v 8.1.1993; vgl. Esambo Kangashe Fn. 9).

Angelo Mobateli, Afin que nul n'en ignore le contenu avant le référendum, nécessité de vulgariser le projet de Constitution, le Potentiel (Kinshasa) v 28.5.2005.

Harmonisiertes Grundgesetz der Konklave, vom Staatspräsidenten am 13.3.1993 verkündet. 


\section{bb) Übergangsgrundgesetz von 1994}

Der Demokratisierungsprozess kam erst wieder in Gang, als die Geberländer ihre Entwicklungshilfe einfroren. Daraufhin einigten sich die streitenden Fraktionen auf eine Zusammenführung der beiden Verfassungsentwürfe zu einem gemeinsamen Text ${ }^{41}$. Darin wurde die Zusammenlegung der konkurrierenden Institutionen mit einer einheitlichen Regierung unter Leitung eines Politikers, welcher einer anderen politischen Strömung als der Staatspräsident angehören soll, sowie einem gemeinsamem Übergangsparlament vorgesehen. 1994 wurde der gemeinsame Verfassungstext in eine Übergangsverfassung umgewandelt, welche die Machtteilung der Konfliktparteien weiter präzisierte ${ }^{42}$. Mobutu behielt den Oberbefehl über die Streitkräfte, während die Regierung die Kontrolle der Zentralbank übernahm. Die Wahl eines neuen Ministerpräsidenten fand auch ordnungsgemäß statt, doch wurden die Parlamentswahlen abgesagt und die Amtszeit des Präsidenten um weitere zwei Jahre verlängert, während sich im Gefolge des Genozids im Nachbarland Ruanda und der massiven Zuwanderung der hieran stark beteiligten Hutu-Milizen über die Grenze auch die Gewalttätigkeiten im Inneren des Kongos wieder verschärften.

Eine Epoche ging für das Land 1997 zu Ende, als Präsident Mobutu mit Unterstützung der Nachbarländer Ruanda und Uganda gestürzt und durch den bis dahin allenfalls als Diamantenschieber bekannten neuen Präsidenten Kabila ersetzt wurde. Dieser wendete sich jedoch rasch von seinen bisherigen Verbündeten ab und näherte sich der alten Elite in Kinshasa an, wobei er sich hauptsächlich auf die südlichen Nachbarn des Kongo stützte.

Wenig später begleitete das kurz gehaltene Verfassungsdekret von $1997^{43}$, welches den Präsidenten mit allen Vollmachten eines autoritären Herrschers ausstattete ${ }^{44}$, den Versuch der Wiederherstellung staatlicher Ordnung ${ }^{45}$. Ein ausführlicher und differenzierter Verfassungsentwurf mit Anklängen an die Luluabourg-Verfassung von 1965 wurde 1998 wurde

Acte constitutionnel harmonisé relatif à la période de transition, Gesetz Nr 93/001 v 2.4.1993 sowie Acte constitutionnel de la transition, Gesetz Nr 94/001 verk am 9.4.1994; Esambo Kangashe (Fn. 9).

Esambo Kangashe (Fn. 9).

43

Verfassungs-Dekret-Gesetz Nr. 003 vom 27.5.1997 über Organisation und Ausübung der Macht in der Demokratischen Republik Kongo.

44

$\mathrm{S}$ v.a. Art 5 und 6, welche dem Präsidenten die Befugnis zur Gesetzgebung, zur Leitung der Verwaltung und der Sicherheitskräfte, zur Ernennung der Richter sowie zum Drucken der Währung verleihen.

45

Esambo Kangashe (Fn. 9). 
zwar von Präsident Kabila sen. gebilligt, von ihm aber nicht der Bevölkerung zur Annahme durch ein Referendum vorgelegt. Im Vorfeld des heraufziehenden neuen Kriegs unternahm die Verfassungsänderung von $1998^{46}$ vielmehr einen letzten Versuch, die in der Realität erodierende Stellung des Präsidenten zumindest auf dem Papier zu festigen ${ }^{47}$. Tatsächlich intensivierte sich aber die Rebellion. Auch ein im Juli 1999 unterzeichnetes Waffenstillstandsabkommen vermochte die Entwicklung nicht in friedlichere Bahnen zu lenken; vielmehr kam es im November 1999 vor allem im Norden und Osten des Landes zu erneuten schweren Gefechten zwischen Regierungstruppen und Rebellen, welche im April des Folgejahres durch einen Waffenstillstand vorläufig beendet werden konnten. Die Lage blieb indes höchst instabil und am 17.1.2001 erlag Staatspräsident Kabila sen. den Folgen eines Attentats.

Wenige Tage darauf übernahm sein damals nur 31 Jahre alter Sohn Joseph Kabila als ein für alle Seiten akzeptabler Kompromiss- und Übergangskandidat die Amtsgeschäfte. Er wurde am 24.1.2001 vom Parlament bestätigt und am 26.1.2001 vereidigt. Er übernahm von seinem Vater ein katastrophales Erbe mit einem zerrütteten Verwaltungs- und Finanzwesen, verfügte über keine eigene Machtbasis in den staatlichen Institutionen und bei den dominierenden ausländischen Mächten (er stützte sich zunächst vor allem auf die relativ schwachen Truppenverbände aus Simbabwe) und konnte nach den Erfahrungen der vorangegangenen Jahre kaum noch auf Transformationshoffnungen zählen. Dennoch nutzte Kabila jun. seine Ausgangsposition der Äquidistanz zu den von ihm vorgefundenen Machtgeflechten und die ihm verfügbaren geringen Handlungsspielräume geschickt, um seine Position rasch zu konsolidieren, internationale Reputation zu gewinnen und den Friedensprozess fortzuführen. So konnten sich im März 2001 die wichtigsten kriegführenden Mächte, also Ruanda, Angola, Simbabwe, Namibia, Burundi und die Demokratische Republik Kongo selber sowie die entsprechenden Rebellenorganisationen auf einen Truppenrückzug und die Stationierung von VN-Einheiten einigen und damit den Weg für die gegenwärtige Transformationsphase frei machen. 
Ein erstes Grundlagendokument der gegenwärtigen Übergangsordnung bildete das Waffenstillstandsabkommen des Jahres 2002 (Accord global et inclusif) ${ }^{48}$. Als Ziele wurden Wiedervereinigung, Befriedung, Wiederherstellung der territorialen Integrität sowie der staatlichen Autorität über das gesamte Staatsgebiet, die nationale Versöhnung, die Bildung einer integrierten Armee, die Abhaltung freier und transparenter Wahlen auf allen Ebenen und die Schaffung einer demokratischen Verfassung sowie die Schaffung neuer politischer Institutionen formuliert (Art II Accord global et inclusif).

$\mathrm{Zu}$ den vordringlichsten Aufgaben dieser Übergangsregierung gehörte die Vorbereitung freier Wahlen innerhalb der Übergangsfrist von zwei Jahren, welche im Falle besonderer Probleme bei der Vorbereitung dieser Wahlen zwei mal um höchsten 6 Monate werden durfte (Art IV Accord global et inclusif). Bis dahin sollte Kabila Staatsoberhaupt bleiben, jedoch mit vier Stellvertretern, die von der bisherigen Regierung, von den beiden größten Rebellenorganisationen, nämlich dem von Uganda unterstützten MLC $^{49}$ und dem von Ruanda unterstützten $\mathrm{RCD}^{50}$ sowie von der zivilen Opposition gestellt werden sollten (Art I (3), V (1) mit Anlage I Accord global et inclusif).

Ferner wurden zwei Gesetzgebungskammern eingerichtet, deren Mitglieder von den Komponenten des Transformationsprozesses zu benennen waren, nämlich ein Abgeordnetenhaus mit 500 Mitgliedern und ein Senat mit 120 Mitgliedern (Art V (2) Accord global et inclusif). Für beide Kammern wurde betont, dass die von jeder Komponente entsandten Vertreter eine ausgewogene regionale Verteilung haben müssen und dass jedem Parlamentarier eine angemessene monatliche Vergütung zusteht. Hauptaufgabe des Abgeordnetenhauses war die Gesetzgebung, Hauptaufgabe des Senates die Ausarbeitung der Staatsverfassung sowie die Mitwirkung bei bestimmten Fragen der Gesetzgebung wie der Wahlgesetzgebung und dem Staatsangehörigkeitsrecht.

Weiter wurden die Unabhängigkeit der Justiz betont (Art V (3) Accord global et inclusif) und fünf Einrichtungen zur Festigung der Demokratie geschaffen, nämlich eine Unabhängige Wahlkommission, eine Hohe Medienbehörde, eine Wahrheits- und Versöhnungs-

Allgemeine und umfassende Vereinbarung über die Transformation in der Demokratischen Republik Kongo (Accord global et inclusif sur la transition en République Démocratique du Congo signé à Prétoria (République d'Afrique du Sud) le 16.12.2002). 
kommission, ein Nationales Observatorium der Menschenrechte sowie eine Ethikkommission zur Bekämpfung der Korruption (Art V (4) Accord global et inclusif). Alle genannten Einrichtungen sollten mit Vertretern der Zivilgesellschaft besetzt werden und unabhängig arbeiten; sie erfüllten ihre Erwartungen allerdings durchweg nicht ${ }^{51}$. Ihre Leiter erhielten den Rang von Ministern. Ferner wurde die Bildung einer nationalen Armee vorgesehen (Art VI Accord global et inclusif) und die Besetzung der Leitungsposten in Staatsunternehmen (Anhang II Accord global et inclusif) geregelt.

\section{bb) Übergangsverfassung 2003}

Auf den Grundlagen des Accord global et inclusif und vor dem Hintergrund anhaltenden internationalen Drucks gelang es den ehemaligen Bürgerkriegsparteien, sich bereits am 1.4.2003 im Rahmen ihres interkongolesischen Dialogs auf eine Übergangsverfassung zu verständigen. Präsident Kabila verkündete diese Übergangsverfassung am 4.4.2003 und ließ sich am 7.4.2003 auf sie vereidigen; daneben blieb der Accord global et inclusif als weiterhin rechtlich relevantes Dokument zu berücksichtigen ${ }^{52}$. Am 30.6.2003 wurde die Übergangsregierung benannt und nach formeller Beendigung der Bürgerkriegshandlungen die 4 Vizepräsidenten eingesetzt. Der Posten für die politische Opposition fiel nicht an einen der Führer der beiden Hauptgruppen, sondern ging an den Vertreter einer Splittergruppe $^{53}$. Auch die Einrichtungen zur Festigung der Demokratie wurden nicht wie vorgesehen von der Zivilgesellschaft, sondern von der alten Regierung und den Rebellenbewegungen dominiert ${ }^{54}$. Am 22.8.2003 nahm das Übergangsparlament seine Tätigkeit auf und am 5.9.2003 wurde ein gemeinsames Generalkommando der bisherigen Rebellenarmeen eingerichtet. Als weiterer Baustein der neuen Ordnung kam am 16.5.2004 die Ernennung der Gouverneure der 11 Provinzen hinzu. Mit deutlichem zeitlichem Abstand folgten als weitere wichtige Schritte am 18.1.2006 die Verkündung der neuen Verfassung und am 6.3.2006 die Ratifikation von 11 internationalen Konventionen einschließlich der Afrikanischen Menschenrechts-Charta sowie der VN-Konventionen zur Bekämpfung von Terroris-

Urteil des als Verfassungsgericht tagenden Obersten Gerichtshofs v 24.2.2006, vgl Donatien Ngandu Mupompa, Interprétation de la Constitution par la Cour suprême de justice, Le Potentiel (Kinshasa) v. 25.2.2006. 
mus, Organisierter Kriminalität und Menschenhandel ${ }^{55}$. Am 11.3.2006 konnte auch das Wahlgesetz ${ }^{56}$ verabschiedet werden.

Dennoch bedeutete dieser Prozess noch kein vollständiges Ende der gewalttätigen Auseinandersetzungen $^{57}$. So wurden am 28.3. und 11.6.2004 Putschversuche aufständischer Einheiten vereitelt, die Stadt Bukavu im Osten des Landes wurde vom 2.-9.6.2004 kurzzeitig besetzt, am 13.8.2004 fielen 160 kongolesische Tutsi-Flüchtlinge einem Massaker in einem Lager im Nachbarland Burundi zum Opfer, wofür burundische Hutu-Milizen, aber auch vom Kongo aus operierende ruandische Hutu-Verbände und kongolesische Maï-Maï-Milizen verantwortlich gemacht wurden. Am 12.12.2004 kam es zu 10-tägigen militärischen Auseinandersetzungen zwischen Regierungssoldaten und Aufständischen im Kanyabayonga-Gebiet der Nord-Kivu-Provinz im Osten des Landes. Am 10.1.2005 wurde eine Demonstration gegen eine mögliche Verlängerung des Transformationsprozesses gewaltsam aufgelöst, mindestens 4 Demonstranten kamen dabei um. Am 25.2., 12.5. und 11.6.2005 fielen Soldaten der internationalen Friedenstruppe Anschlägen zum Opfer, vom 2.-11.6.2005 wurden Freiwillige der Hilfsorganisation Ärzte ohne Grenzen von Aufständischen entführt. Außerdem brachen alte ethnische Konflikte, welche unter der Diktatur Mobutu und anschließend den ausländischen Besatzungstruppen weitgehend unterdrückt worden waren, erneut auf. So kam es im Ituri-Gebiet im Nordosten des Landes zu gewaltsamen Auseinandersetzungen zwischen Angehörigen der Volksgruppen der Lendu und der Hema. Andererseits vermeldeten die Vereinten Nationen fast zeitgleich, dass sie im Hauptkonfliktgebiet Ituri im Osten des Landes die Aufständischen fast vollständig entwaffnet habe $^{58}$.

Auch die Übergangsverfassung von 2003 schuf in den Grundfragen der künftigen Ausgestaltung des Staatswesens wenig Klarheit und konzentrierte sich vor allem auf eine effektive Machtbalance der beteiligten Konfliktparteien ${ }^{59}$. Neben der Umsetzung der Beschlüsse

Vgl Art III (3) Accord global et inclusif; N.N., Plénière de l'Assemblée nationale, Le Potentiel (Kinshasa) v 7.3.2006.

Gesetz Nr 06/006 v 9.3.2006 über die Organisation der Präsidial-, Parlaments-, Provinz-, Stadt und Gemeindewahlen mit Erlass Nr 004/CEI/BUR/06 v 9.3.2006; vgl. Médard Muyaya, Parlament: les deputés et sénateurs ont adopté la loi éléctorale, Le Potentiel v 23.2.2006.

Cheick Yérim Seck, En attendant les élections, Jeune Afrique v 25.7.2004.

58 MONUC, La RDC depuis le lancement de la transition en 2003, Le Potentiel (Kinshasa) v 20.6.2005. tion, Le Potentiel (Kinshasa) v. 31.5.2005 bezeichnet diesen Zustand als Diktatur der Bürgerkriegsparteien. 
des Accord global et inclusif basierte dieser Text vor allem auf dem Entwurf von 1998 sowie der Luluabourg-Verfassung von 1964.

\section{Prozess der Verfassungsgebung 2005}

Auch der Prozess der Verfassungsgebung verlief trotz der mit der Übergangsverfassung bereits geleisteten Vorarbeit keineswegs reibungsfrei. Einerseits gab es lebendige Diskussionen nicht nur in den Parlamentskammern, sondern zumindest in der Hauptstadt Kinshasa auch eine kurze, aber intensive gesellschaftliche Debatte ${ }^{60}$. Andererseits drohte der Übergangsprozess immer wieder ins Stocken zu geraten, so dass schon bald die Frage seiner Verlängerung zu einem zentralen Punkt sowohl der nationalen Diskussion im Kongo als auch mit den internationalen Gebern wurde ${ }^{61}$. Gemäß der Übergangsverfassung war der Entwurf vom Senat zu erstellen (Art 98) und die endgültige Verfassung vom Abgeordnetenhaus zu verabschieden (Art 104 (2)). Der Senat setzte im Herbst 2003 einen entsprechenden Ausschuss ein. Dieser begab sich alsbald in der Stadt Kisangani mit internationaler, vor allem belgischer Beratung in Klausur und startete auf dieser Grundlage im Februar 2004 eine landesweite Befragung ${ }^{62}$. Nach "langen und schmerzhaften" Beratungen, wie es der Senatspräsident formulierte ${ }^{63}$, und nicht zuletzt internationalem Druck auf eine zügige Verabschiedung konnte der Senat den Vorentwurf ${ }^{64}$ schließlich am 17.3.2006 verabschieden und am 23.3.2005 an das Abgeordnetenhaus weiterreichen ${ }^{65}$. Als Leitgedanken wurden bei dieser Gelegenheit die Kompromissfindung, die Stabilität der künftigen Institutionen, der Schutz der Menschenrechte, der persönliche und gemeinschaftliche Fortschritt sowie

Vgl. etwa Constitution de la 3ème République: 53 grands principes pour compléter l'avant-projet du Sénat, Le Phare (Kinshasa), v 21.2.2005; Dominique Mukenza, Avant projet de la future constitution: l'âge du président divise les Sénateurs; $J p$ Mbelu, Démocratie représentative et consensus maximal, beide jeweils Le Phare (Kinshasa) v 25.2.2005; Espérance Tshibuabua, Du 24 au 26 février 2005, les Ongs congolaises réflêchissent sur l'impunité en Rdc, Le Potentiel (Kinshasa v 25.2.2005); Angelo Mobateli (Fn. 28); Jean-N'saka, Le Peuple congolais roulé dans la farine? Le Phare (Kinshasa) vom 31.1.2005; Rich Ngapi (Fn. 59).

Jean-N'saka (Fn. 60); MONUC, La RDC depuis le lancement de la transition en 2003, Le Potentiel (Kinshasa) v 20.6.2005; A propos du 30 juin 2005, Joseph Kabila consulte, Le Potentiel (Kinshasa) v 26.4.2005.

Le Sénat transmet à l'Assemblée nationale la mouture du texte de l'avant-projet de la Constitution de la $3^{\text {ème }}$ République, Digitalcongo v 24.3.2005.

Le texte de l'avant-projet de la Constitution, Le Phare (Kinshasa) v 21.3.2005.

Le Sénat transmet à l'Assemblée nationale la mouture du texte de l'avant-projet de la Constitution de la 3ème République, Digitalcongo v 24.3.2005; GK/DM, L'avant-projet de la Constitution transmis au Sénat, Le Phare (Kinshasa) v 24.3.2005. 
die nationale Eintracht und Einheit herausgestellt ${ }^{66}$. Eine knappe Woche später begann das Abgeordnetenhaus seinerseits mit der Prüfung des vom Senat ausgearbeiteten Vorentwurfs. Im Zuge der stürmischen Debatten wurden über 700 Änderungsanträge meist individuell von einzelnen Abgeordneten eingebracht und in Ausschüssen und Plenum heftig debattiert $^{67}$. Dabei kam es noch einmal zu wesentlichen inhaltlichen Änderungen gegenüber dem Vorentwurf des Senats. Dabei wurde in wesentlichen Fragen ein Kompromiss zwischen den wichtigsten politischen Strömungen erreicht, aber auch die Handschrift des gegenwärtigen Staatspräsidenten deutlich.

Besonders intensiv diskutiert wurde das Mindestalter der Kandidaten für das Amt des Staatspräsidenten, welches schließlich auf 30 Jahre festgesetzt wurde, so dass eine erneute Kandidatur des derzeitigen Amtsinhabers Kabila unproblematisch ist.

In einem zweiten Punkt erreichte dagegen die Ruanda nahestehende RCD einen Teilerfolg, indem einerseits die Rechte ethnischer Minderheiten besonders betont wurden, während andererseits die ruandaphonen Banyamulenge, denen auch der von der RCD gestellte Vizepräsident Ruberwa angehört, als besonders unter Druck stehende Minderheit nicht ausdrücklich erwähnt wurden ${ }^{68}$. Institutionell wurde die Stellung der Regierung gegenüber dem Staatspräsidenten gestärkt, welcher gleichwohl eine wichtige Rolle bei der Festlegung der Regierungspolitik behält. Weiter wurde die Abhaltung von Wahlen in Gebietskörperschaften präzisiert. Von der Aufzählung der durch Wahlen zu bestimmenden Verwaltungsleiter wurde allerdings die unterste Ebene ausgenommen, also alle Gemeinden mit Ausnahme der Hauptstadt Kinshasa, die Sektoren sowie die chefferies (Sitze lokaler, traditioneller Führer) ${ }^{69}$. Ferner wurde die Todesstrafe abgeschafft. Weitere Punkte betrafen die Aufgabenteilung zwischen zentraler und regionaler Ebene der Staatsgewalt, die Gewaltenteilung, die Auflösung der Einrichtungen zur Unterstützung der Demokratie, der Schutz von Teilen der Verfassung vor späteren Verfassungsänderungen sowie die Neutralität der Polizei $^{70}$. Mit einem wesentlichen Teil der Änderungen wurde auch Forderungen der EU als wichtigstem Finanzgeber entsprochen ${ }^{71}$.

Le Sénat transmet à l'Assemblée nationale la mouture du texte de l'avant-projet de la Constitution de la $3^{\text {ème }}$ République, Digitalcongo v 24.3.2005.

Médard Muyaya, Assemblée nationale: fin de l'examen du projet de Constitution, Le Potentiel (Kinshasa) v 14.5.2005 ; Devant 700 amendements, l'avant-projet de Constitution dans l'impasse, Le Potentiel (Kinshasa) v 5.5.2005. N.N., Constitution: Le Pprd joue et gagne, Le Potentiel v. 16.5.2006.

Vgl N.N., Projet de Constitution de la République démocratique du Congo: les deputés congolais suppriment les élections locales, Le Potentiel (Kinshasa) v 16.5.2005. 
Die Annahme erfolgte weniger als 2 Monate später am 16.5.2005. Von den 500 Abgeordneten waren bei der Abstimmung 361 anwesend, von welchen 348 (entsprechend 65,6\% der Zahl der gesetzlichen Mandatsträger) den Entwurf billigten, 5 (entsprechend $1 \%$ ) dagegen stimmten und 8 (entsprechend $1,6 \%$ ) sich enthielten ${ }^{72}$.

Am 14.6.2005 wurde mit der Annahme das Gesetz über die Durchführung von Volksabstimmungen eine weitere wichtige Voraussetzung für eine Fortsetzung des Transformationsprozesses geschaffen und am Folgetag - die geltenden Fristen fast vollständig ausschöpfend - die Aufschiebung des Endtermins der Übergangszeit bis zum 31.12.2005 mit einer nochmaligen Verlängerbarkeit um weitere 6 Monate, also bis zum 30.6.2006, beschlossen $^{73}$.

Nach einer kurzen Phase öffentlicher Diskussion wurde im Dezember 2005 eine reguläre Verfassung in einer von unabhängigen Beobachtern als im wesentlichen frei und fair charakterisierten $^{74}$ Volksabstimmung mit deutlicher Mehrheit angenommen ${ }^{75}$. Laut dem amtlichen Endergebnis beteiligten sich 61,97 \% der rd. 25 Mio. registrierten Wähler an der Abstimmung $^{76}$. Von diesen stimmten $84,31 \%$ der Vorlage zu, 15,69\% stimmten dagegen. Besonders niedrig war die Zustimmung in der Hauptstadt Kinshasa, wo nur eine knappe Mehrheit der Wähler den Text billigten. Dies wird teils auf die Kampagne des führenden Oppositionspolitikers Tshisekedi für einen Wahlboykott ${ }^{77}$ sowie auf eine verbreitete Enttäuschung in der lebendigen Zivilgesellschaft über eine unzureichende Verbreitung und Diskussion des Entwurfs zurückgeführt. Nach seiner Annahme wird der neue Grundlagentext inzwischen aber auch von bisherigen Kritikern als Grundlage für die weitere Transformation akzeptiert ${ }^{78}$. Die damit verbundene Ausrufung einer „III. Republik“ bekundet die Hoffnung, ein neues Kapitel in der Geschichte des Landes aufzuschlagen.

Die neue Verfassung stärkt im Sinne einer Machtbalance u.a. die Befugnisse des Ministerpräsidenten sowie der regionalen Körperschaften. Sie soll auch die Grundlage für eine Fortsetzung des Transformationsprozesses mit Wahlen zu den wichtigsten Staatsorganen im

DR Congo parliament adopts new constitution, AFP v 13.5.2005.

N.N., Prolongation de la transition: le parlement n'a pas surpris, Le Potentiel (Kinshasa) v 20.6.2005.

74 Vgl Vereinten Nationen, Le Secrétaire général salue la promulgation de la Constitution, allafrica.com v 22.2.2006.

75 Vgl UN-IRIN v 12.1.2006, Les Congolais plébiscitent la nouvelle constitution.

76

UN-IRIN v 12.1.2006, DRC: Voters Give New Constitution Overwhelming Support.

77

78

UN-IRIN v 12.1.2006, Les Congolais plébiscitent la nouvelle constitution.

Vgl UN-IRIN v 12.1.2006, Les Congolais plébiscitent la nouvelle constitution. 
Laufe des Jahres 2006 bilden ${ }^{79}$. Noch kommt es aber immer wieder zu militärischen Aktionen ausländischer Mächte und internen Auseinandersetzungen, sind weit mehr als 2 Mio. Einwohner intern vertrieben ${ }^{80}$, ist die Infrastruktur weitgehend zerstört ${ }^{81}$ und liegt das Bruttoinlandsprodukt mit knapp 5 Mrd. US-\$ sogar unter dem Niveau von $1958^{82}$.

\section{Struktur der neuen Verfassung}

Die Verfassung lehnt sich eng an ihre Vorläuferin aus dem Jahre 2003 an. Insgesamt ist die Verfassung um 31 Artikel gewachsen. Die zusätzlichen Artikel betreffen insbesondere die Grundrechte, die Einführung eines Abschnitts über Grundpflichten, die Regelungen über das Parlament, den Finanzausgleich, die öffentliche Verwaltung, Lokalverwaltungen, traditionelle Verwaltungen, den Verfassungsgerichtshof sowie die Änderung der Verfassung. Für diese Ergänzungen wurden vor allem der Entwurf von 1998 sowie die LuluabourgVerfassung von 1965 herangezogen. Die neue Verfassung folgt wie bereits ihre Vorläuferin dem auch in den meisten anderen frankophonen Ländern zu beobachtenden Muster und enthält nach einem einleitenden Abschnitt mit Staatsgrundsätzen und der im Kongo besonders umstrittenen Frage der Staatsangehörigkeit einen Grundrechtsabschnitt. Die Regelungen der staatlichen Institutionen umfassen Regelungen zum Amt des Staatspräsidenten, zur Regierung, zu den beiden parlamentarischen Kammern und zur Gesetzgebung sowie zum Justizwesen. Es folgen Regelungen zum Finanz- und Haushaltswesen, zur Zentralbank, zum Rechnungshof sowie zum innerstaatlichen Finanzausgleich. Weitere Regelungen betreffen die Sicherheitsorgane, die öffentliche Verwaltung, die Lokalverwaltungen und die traditionellen Autoritäten. Als Sonderinstitutionen werden der Wirtschafts- und Sozialausschuss, die unabhängige Wahlkommission und die Hohe Medienbehörde geregelt. Am Ende stehen Bestimmungen zu internationalen Vereinbarungen, zur Verfassungsänderung sowie die Übergangs- und Schlussbestimmungen.

Vgl Kabila, " I am optimistic that elections can be held this year", International Herald Tribune v 1.2.2005, S $18 \mathrm{ff}$.

80 Stand Oktober 2004 nachdem die Zahl ein Jahr zuvor noch um weit über 1 Mio höher gelegen hatte, vgl Norwegian Refugee Council, Profile of Internal Displacement: Democratic Republic of the Congo, Genf 2004, S 50.

81

Erste Anzeichen der Besserung wie die Wiedereröffnung der Bahnlinie zwischen Lubumbashi und Kindu im Osten dürfen über den riesigen Investitionsbedarf nicht hinwegtäuschen vgl Derrick, Linking Congo: Air service is up, but roads are needed, International Herald Tribune v 1.2.2005, S 21.

82

Vgl L'économie congolaise dans la cyclone, Le Potentiel (Kinshasa) v 23.2.2005. 


\section{Staatsgrundsätze}

Im Einleitenden Kapitel regelt die Verfassung eine elementarer Grundfragen. Hierzu gehören insbesondere die Staatsgrundsätze, welche den Bestand des Kongo in seinen Grenzen im Zeitpunkt der Unabhängigkeit festschreiben und ihn als einen Rechtsstaat, unabhängig, souverän, einig und unteilbar, sozial, demokratisch und laizistisch (Art 1 (1) Verf(2005)) kennzeichnet sowie Französisch als Amtssprache (Art 1 (7) Verf(2005)) und Kikongo, Lingala, Swahili und Tshiluba (Art 1 (8) Verf(2005)). Ferner werden die Regionaleinteilung (Art 2, 3 Verf(2005)), die Ausübung der Staatsgewalt durch das Volk (Art 5 Verf(2005)) ${ }^{83}$, der politische Pluralismus (Art 6-8 Verf(2005)), die Staatssouveränität über Bodenflächen (Art $9 \operatorname{Verf}(2005))^{84}$ und die Staatsangehörigkeit (Art $\left.10 \operatorname{Verf}(2005)\right)^{85}$ behandelt. Eine verfassungsrechtliche Gewährleistung institutioneller Gewaltenteilung existiert bislang lediglich im Hinblick auf eine Machtbalance der ehemaligen Bürgerkriegsparteien. Sie wird auch in der neuen Verfassung nicht in allgemeiner Form thematisiert (wohingegen der Art 76 des Entwurfs von 1998 dies in ausdrücklicher Form vorgesehen hatte), findet sich jedoch u.a. in Bestimmungen zum Verbot des imperativen Mandats in den beiden Parlamentskammern ${ }^{86}$, Inkompatibilitätsregelungen ${ }^{87}$ und Bestimmungen zur Unabhängigkeit der Justiz ${ }^{88}$.

\section{Grund- und Teilhaberechte}

\section{a) Lage der Menschenrechte}

Der Schutz von Grundrechten ist nicht gewährleistet ${ }^{89}$. Zwar ist eine wachsende politische und gesellschaftliche Toleranz zu beobachten. Nach Jahren der Gewalttätigkeit sind jetzt auch wieder friedliche Demonstrationen möglich ${ }^{90}$. Leider bleibt diese Verbesserung der Lage bislang nur auf die von der Regierung kontrollierten Gebiete beschränkt. Überall bleibt die Stabilisierung des Rechtswesens weiterhin eine unverzichtbarer Voraussetzung

S.u. Ziff. 17.

84

85

S.u. Ziff. 30.

S.u. Ziff. 9.

86

87

u. Ziff. 14

S.u. Ziff.10.

S.u. Ziff. 18.

89

Vereinigtes Königreich, Home Office, Immigration and Nationality Directorate, Democratic Republic of Congo - Country Report, London 2004, Ziff Ziff 6.1 ff.

90

Première manifestation pacifique à Kinshasa depuis la transition, Xinhua v 22.3.2006. 
für weitere Verbesserungen. Es gibt besonders in der Hauptstadt Kinshasa eine lebendige, aber schlecht organisierte Zivilgesellschaft. Neben den Kirchen ist der Unternehmerverband die einzig nennenswerte Organisation der Zivilgesellschaft. Ebenso gibt es ein reichhaltiges Medienangebot mit rund 165 Periodika, von welchen allerdings nur rund 20 tatsächlich regelmäßig erscheinen; darunter 8 Tageszeitungen ${ }^{91}$. Die Auflage aller Erzeugnisse ist gemessen an der Bevölkerungszahl geringfügig; keines dieser Periodika übersteigt 2.500 Exemplare. Ferner senden 25 Radio- und 22 Fernsehstationen, von denen jeweils die beiden bedeutendsten Kanäle in staatlicher Hand sind. Trotz dieser Vielfalt herrscht keine Pressefreiheit $^{92}$. Regierungskritische Berichterstattung kann sich für den Autor als lebensgefährlich erweisen ${ }^{93}$; Misshandlungen und Festnahmen missliebiger Journalisten sind verbreitet $^{94}$. Ein weiteres Problem besteht darin, dass unterbezahlte Journalisten für ihre redaktionelle Berichterstattung immer häufiger ein Honorar von ihrem Gesprächspartner erwarten.

Die mit großen Erwartungen eingeführten Einrichtungen zur Stärkung der Demokratie haben die hohen Erwartungen nicht erfüllen können. Die durch die Verfassung von 2003 nach südafrikanischem Vorbild eingerichtete Wahrheits- und Versöhnungskommission zur Aufklärung der Bürgerkriegsverbrechen hat alle Erwartungen enttäuscht ${ }^{95}$ und ist in der Verfassung von 2005/06 nicht mehr enthalten. Als Gründe hierfür werden deren Zusammensetzung aus Vertretern der ehemaligen Bürgerkriegsparteien sowie insbesondere die Parteilichkeit ihres Vorsitzenden angeführt ${ }^{96}$. Ähnlich konnte auch das neu geschaffene Menschenrechtsobservatorium keine nennenswerten Beiträge zur Stärkung der Menschenrechte leisten. Gleiches gilt für das Menschenrechtsobservatorium. Aufgrund seiner Besetzung seiner heterogenen Besetzung mit Vertretern der Bürgerkriegsparteien spiegelt es mehr die politischen Präferenzen seiner Mitglieder als ein Bemühen um die Wahrung der Menschenrechte $^{97}$. Auch diese Institution wird von der Verfassung von 2005/06 nicht mehr erwähnt. Die Hohe Medienbehörde hat es weder vermocht, der Aufstachelung zum Hass gegen Minderheiten durch Organe der Medien noch deren Kontrolle durch der Regierung

Vereinigtes Königreich, Home Office, Immigration and Nationality Directorate, Democratic Republic of Congo - Country Report, London 2004, Ziff Ziff 6.9.

Vereinigtes Königreich, Home Office, Immigration and Nationality Directorate, Democratic Republic of Congo - Country Report, London 2004, Ziff Ziff 6.8 ff. Journaliste en danger, JED Investigation prompts Death Threats, Kinshasa, Pressemitteilung v 28.2.2006. Republic of Congo - Country Report, London 2004, Ziff Ziff 6.10. 
nahe stehende Kräfte wirksam gegenzusteuern ${ }^{98}$. Weitere Kritikpunkte betreffen die Ausschaltung eines wirksamen Wettbewerbs durch Harmonisierung der Tarife und das Fehlen von Regelung zur Nutzung der Medien durch politische Kräfte.

Ein wesentlicher Baustein für den internen Transformationsprozess war der Beitritt zu insgesamt 11 internationalen Konventionen einschließlich der Afrikanischen Menschenrechts-Charta von VN-Konventionen zur Bekämpfung von Terrorismus, Organisierter Kriminalität und Menschenhandel ${ }^{99}$.

\section{b) Regelungen und Gewährleistungen der neuen Verfassung}

Der Anteil der Grundrechtsartikel wurde gegenüber der Übergangsverfassung deutlich ausgedehnt. Gegenstand von Diskussionen war vor allem die Todesstrafe, deren Beibehaltung nach einer breiten Überzeugung von Parlamentariern zur Sicherung von Recht und Ordnung notwendig schien und auch in dem vom Senat verabschiedeten Vorentwurf (Art 15 (4)) wie bereits in der Verfassung von 2003 (Art 15 (4)) ausdrücklich verankert war ${ }^{100}$. Auf internationalen Druck wurde die entsprechende Bestimmung dann aber aus dem endgültigen Text gestrichen, so dass sich die Abschaffung der Todesstrafe zwar nicht ausdrücklich, aber doch im Rückschluss aus der grundrechtlichen Gewährleistung des Lebens ergibt.

Als bürgerliche und politische Rechte regelt die Verfassung u.a. den Schutz vor sexueller Gewalt (Art 15 Verf(2005)), der körperlichen Unversehrtheit (Art 16, 18 (4) Verf(2005)), der persönlichen Freiheit (Art 17 Verf(2005)), der Meinungs-, Gewissens- und Religionsfreiheit (Art 22 Verf(2005)), der Freiheit der Meinungsäußerung (Art 23 Verf(2005)) und der Massenmedien. Ferner wird Unabhängigkeit und gleicher Zugang zu den staatlichen Massenmedien vorgeschrieben (Art 24 (4) Verf(2005)). Weitere Grund- und Menschenrechte sind die Versammlungs- (Art 25 Verf(2005)), Demonstrations- (Art 26 Verf(2005)) und Vereinigungsfreiheit (Art 37 Verf(2005)), das Petitionsrecht (Art 27 Verf(2005)), das Recht zur Verweigerung illegaler Befehle (Art 28 Verf(2005)), der Unverletzlichkeit der Wohnung (Art 29 Verf(2005)), der Privatsphäre sowie des Post- und Fernmeldegeheimnisses (Art 31 Verf(2005)), die Freizügigkeit (Art 30 Verf(2005)) sowie das Asylrecht insbesondere für wegen ihrer Meinung, ihres Glaubens, ihrer rassischen, stammesmäßigen,

98

99

100

100

Médard Muyaya/A. Tshiambi, Avant-projet de Constitutio
tient la peine capitale, Le Potentiel (Kinshasa) v. 9.3.2005.
Langana et al.(Fn. 51), S. 1, 6 ff. Vgl Art III (3) Accord global et inclusif; N.N., Plénière de l'Assemblée nationale, Le Potentiel
(Kinshasa) v 7.3.2006. 
ethnischen oder sprachlichen Zugehörigkeit oder wegen ihres Einsatzes für Demokratie und Menschenrechte Verfolgte (Art 33 Verf(2005)). Gegenüber dem Senatsentwurf gestärkt wurde das Recht auf Versammlungsfreiheit. Im Falle einer Versammlung in öffentlichen Räumen oder unter freiem Himmel braucht die schriftliche Anmeldung nicht mehr mindestens 48 Stunden im voraus erfolgen, auch ist die zunächst vorgesehene zivilrechtliche Haftpflicht der Organisatoren für Ordnungsverstöße und die Verletzung von Rechten Dritter (Art 25 des Entwurfs) entfallen.

Wirtschaftlich relevant sind das Recht auf Eigentum und Schutz vor Enteignungen (Art 34 Verf(2005)), auf wirtschaftliche Betätigung (Art 35 Verf(2005)), auf Arbeit (Art 36 Verf(2005)) sowie auf gewerkschaftliche Betätigung (Art 38 Verf(2005)) und Streik (Art 39 Verf(2005)); eine Beschränkung des Streikrechts ist nur durch Gesetz auf den Gebieten der nationalen Verteidigung bzw Sicherheit oder hinsichtlich öffentlicher Dienstleistungen von vitalem Interesse (Art $39 \operatorname{Verf(2005)).}$

Der Katalog kultureller und sozialer Teilhaberechte beschränkt sich auf einen kurzen Katalog elementarer gesellschaftlicher Herausforderungen. Genannt werden das Recht auf Kinder- und Jugendschutz (Art 41-43 Verf(2005)), auf Kultur und geistiges Eigentum (Art 46 Verf(2005)), auf Gesundheit und Lebensmittelsicherheit (Art 47 Verf(2005)), auf angemessene Unterkunft, auf Zugang zu Trinkwasser und Strom (Art 48 Verf(2005)) sowie auf Schutz alter und behinderter Menschen (Art 49 Verf(2005)). Auch bei diesen Regelungen bleibt allerdings deren praktische Durchsetzung eine offene Frage. Eher familienrechtlicher Natur sind das Recht auf Freiheit der Eheschließung und auf Zusammenhalt in der Familie (Art 40 Verf(2005)),

Darüber hinaus widmet die Verfassung der Gleichbehandlung seiner Bürger in unterschiedlichen Bestimmungen besondere Aufmerksamkeit. So wird der allgemeine Gleichheitsgrundsatz gleich doppelt formuliert (Art 11, 12 Verf(2005)) und um spezifische Diskriminierungsverbote im Hinblick auf Bildung und den Zugang zu staatlichen Ämtern (Art 13, 14, 45 (3)), zum Schutz von Frauen (Art 15) sowie zum Schutz von Ausländern (Art 11, 32, 35 (1), 50 (2)) ergänzt; zweifelhaft ist allerdings die Bedeutung des nur in Art 50 (2) aufgeführten Reziprozitätserfordernisses im Falle von Ausländern. In diesen vielfältigen Regelungen spiegelt sich nicht nur eine Nachwirkung der Segregationspolitik der ehemaligen Kolonialmacht Belgien, sondern vor allem auch die Befürchtung von Minderheiten, in einer Demokratie durch Mehrheitsentscheidungen in ihren Rechten verkürzt zu werden.

Neben den individuellen Grundrechten wird auch eine Gruppe kollektiver Grundrechte geregelt. Dazu gehören die friedliche Koexistenz ethnischer Gruppen und der Schutz von Minderheiten (Art 51 Verf(2005)), das Recht auf Frieden (Art 52 Verf(2005)), auf gesunde Umwelt (Art 53-55 Verf(2005)), auf Teilhabe am natürlichen Reichtum (Art 56-59 Verf(2005)). Offen gelassen wird dabei allerdings, wie diese Rechte anders als durch 
Stimmabgabe bei demokratischen Wahlen geltend gemacht werden können. Ihnen dürfte deshalb eher eine Wirkung vergleichbar von Staatszielbestimmungen zukommen.

Neben den Grundrechten kennt die Verfassung auch ein Kapitel zu den Grundpflichten der Bürger. Zu diesen zählen insbesondere die Kenntnis und Beachtung der Gesetze (Art 62 Verf(2005)), zur Landesverteidigung und Wehrpflicht (Art 63 Verf(2005)), zur Verhinderung von Grundrechtsverletzungen und von Staatsstreichen (Art 64 Verf(2005)), zur Steuerzahlung (Art 65 Verf(2005)), zur Vermeidung der Diskriminierung und zu Toleranz gegenüber Mitbürgern (Art $66 \operatorname{Verf(2005))}$ sowie zum Schutz öffentlichen Eigentums und zur Achtung privaten Eigentums (Art 67 Verf(2005)). Auch hier bleibt die tatsächliche Wirkung dieser Normen abzuwarten, beispielsweise ob sich aus dem Diskriminierungsverbot auch zivilrechtlich oder arbeitsrechtlich einklagbare Ansprüche im Verhältnis der Bürger untereinander ableiten lassen.

Von den menschenrechtsrelevanten Einrichtungen zur Stärkung der Demokratie wird nur

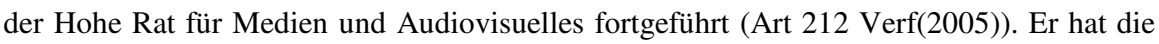
Einhaltung der Presse- und Medienfreiheit sowie den gleichen Zugang zu sichern. Einzelheiten sind durch ein Organgesetz zu regeln.

Rechtsstaatlich unbefriedigend ist, dass im Falle des Ausnahmezustandes ${ }^{101}$ auch die Grundrechte eingeschränkt werden können (Art 61 Verf(2005)). Ausgenommen von diesen Beschränkungsmöglichkeiten sind nur das Recht auf Leben, das Verbot der Folter, der grausamen oder unmenschlichen Behandlung, der Versklavung, der Grundsatz "nulla poena sine lege" und das Recht auf Verteidigung und Rechtsmittel, das Verbot der Freiheitsentziehung wegen Schulden sowie die Meinungs- (nicht: Meinungsäußerungs-), Gewissensund Religionsfreiheit. Dagegen ist eine dauerhafte Senkung des derzeitigen Niveaus des Schutzes der persönlichen Grundrechtsschutzes selbst im Wege der Verfassungsänderung ist ausgeschlossen (Art 220 (2) Verf(2005)). Aus dieser Formulierung dürfte abzuleiten sein, dass eine Ersetzung der bisherigen Normen durch andere mit einem gleichwertigen oder höheren Schutzniveau ebenso zulässig wäre wie eine Absenkung des Schutzniveaus bei den wirtschaftlichen, kulturellen und sozialen sowie bei den kollektiven Grundrechten.

\section{Staat und Religion}

Nach schwierigen Zeiten unter dem Mobutu-Regime haben sich die christlichen Kirchen im Zuge des Transformationsprozesses neues Ansehen zu schaffen vermocht. Weit mehr als die Hälfte der Bevölkerung sind Christen, darunter befinden sich rund $50 \%$ Katholiken, 
rund $20 \%$ Protestanten sowie rund 10\% Kimbangisten (eine bedeutende afrikanische Kongregation $\left.^{102}\right)^{103}$. Außerdem gibt es eine bedeutende islamische Gemeinde (rund 10\%) sowie kleinere jüdische und griechisch-orthodoxe Gemeinschaften. Quer dazu ist der traditionelle Naturglaube nach wie vor in breiten Schichten der Bevölkerung lebendig ${ }^{104}$. Die christlichen Kirchen stellen wichtige Sozial-, Bildungs- und Gesundheitsdienstleistungen zur Verfügung. Gesellschaftlich haben sie eine integrative Wirkung und fördern den gesellschaftlichen Diskurs der intellektuellen Elite des Landes.

Die Verfassung wird diese wichtige Rolle der Religion kaum sichtbar. Gleich in der ersten Bestimmung wird klargestellt, dass der Kongo ein laizistischer Staat ist (Art 1 (1) Verf(2005)). Eine Diskriminierung aus religiösen Gründen ist insbesondere beim Zugang zu Bildungseinrichtungen und zu Staatsämtern verboten (Art 14, 45 (3) Verf(2005)). Die Freiheit der Religionsausübung wird gewährleistet (Art 22 Verf(2005)). Ausgeschlossen sind lediglich Beschuldigungen wegen Hexerei (Art 41 (4) Verf(2005)).

\section{Staatsgebiet \\ a) Entwicklung}

Das Land ist mit einer Fläche von über 2,3 Mio $\mathrm{km}^{2}$ das drittgrößte Afrikas. Es grenzt ähnlich wie Deutschland an insgesamt 9 Nachbarn, nämlich im Norden an die Zentralafrikanische Republik und den Sudan, im Osten an Uganda, Ruanda, Burundi und den Tanganjikasee an der Grenze zu Tansania, im Süden an Sambia, im Südwesten an Angola und im Nordwesten an die Republik Kongo. Ferner hat es einen etwa $40 \mathrm{~km}$ langen Streifen entlang der Atlantikküste; der die angolanische Provinz und Exklave Cabinda vom Rest Angolas abtrennt. Die Ausdehnung in Ost-West- bzw. Nord-Süd-Richtung beträgt jeweils rund $2.000 \mathrm{~km}$.

102 Die Kisangistische Kirche entstand als authochtone kongolesische Kirche aufgrund des charismatischen Wirkens ihres Gründers Simon Kimbangu in den frühen 20er Jahren des 20. Jahrhunderts. $\mathrm{Zu}$ den Glaubensüberzeugungen dieser protestantischen Kongregation gehört eine strikte Auslegung der Bibel, kompromisslose Ablehnung von Zauberei und naturreligiöser Mythologie sowie Verbot der Polygamie. In der Mobutu-Ära war die Kirche eng mit der politischen Führung verbunden.

103 U.S Department of State, International Religious Freedom Report 2004, DR Congo, Washington 2004, Sec. I.

104 Vgl Bayona-ba-Meya Muna Kimvimba, Le juge zairois et la sorcellerie, Recueil Penant 1978, S $303 \mathrm{ff}$. 
Die enorme Größe und der große Rohstoffreichtum haben immer wieder Abspaltungstendenzen gefördert, welche im Falle der Sezession Katangas für gewisse Zeit auch erfolgreich schienen ${ }^{105}$. Hinzu kommt die Einmischung der Nachbarstaaten und der von diesen unterstützten Milizen, welche das Land während des Bürgerkriegs in Einflusszonen unterteilten. So blieb die rechtliche Absicherung der staatlichen Einheit ein wichtiges Anliegen aller Verfassungen.

\section{b) Verfassungsrechtliche Regelung}

Auch die Verfassung von 2005 widmet sich dieser Problematik eingehend. So bestimmt bereits Art 1 (1) die Einheit und Unteilbarkeit des Staates. Politische Parteien müssen die Einheit des Landes respektieren (Art 6 (4)). Jede Verwaltungsstelle hat die Verpflichtung, die Einheit und territoriale Integrität des Landes zu wahren; die Verletzung dieser Pflicht ist Hochverrat (Art 63, 165) Dies gilt in besonderem Maße für den Staatspräsidenten als Garanten der territorialen Integrität (Art 69 (3) S 2), der auf die Wahrnehmung dieser Pflichten auch besonders vereidigt wird (Art 74). So erscheint es keineswegs als ausgeschlossen, dass die Unterzeichnung eines Grenzabkommens bereits als Hochverrat gewertet werden könnte. Andererseits unterstreicht die Fülle der einschlägigen Bestimmungen nur die Labilität des gegenwärtigen Staatsgebildes.

\section{Staatsangehörigkeit}

Angesichts der ethnischen Vielfalt in aus der Kolonialzeit überkommenen Grenzen und umfangreicher Migrationsbewegungen, welche sich in der Region schon immer vollzogen haben und sich im Zuge der wirtschaftlichen Entwicklungen und militärischer Konflikte verstärkt haben, existiert in der DR Kongo kein homogenes Staatsvolk ${ }^{106}$. Das Staatsangehörigkeitsrecht spielte in den aktuellen Konflikten eine zentrale Rolle, da es u.a. in wirtschaftlicher (Grundbesitz) und politischer Hinsicht (aktives und passives Wahlrecht) erhebliche Hebelwirkungen entfaltete. Es wurde deshalb nicht primär als ein Instrument zum Ausgleich der Interessen der beteiligten Individuen, sondern vor allem als eine zentrale Frage der Macht im Inneren und der Verfolgung der staatlichen Interessen nach außen wahrgenommen. So gehörte 2005 die Neuregelung des Staatsangehörigkeitsrechts zu den Grundvoraussetzungen, um im Folgejahr die Wählerregistrierung für die vereinbarten Wahlen durchführen zu können.

105 S. o. Ziff 2. 
Von zentraler praktischer Bedeutung ist noch heute die Frage des Erwerbs der Staatsangehörigkeit bei Staatsgründung ${ }^{107}$. Im Mittelpunkt der Diskussion stehen die Angehörigen der vor allem im Nachbarland Ruanda ansässigen Bevölkerungsgruppen der Hutu, Twa und vor allem der Tutsi, welche meist unter dem Sammelbegriff der Banyarwanda ${ }^{108}$ zusammen gefasst werden und deren Staatsangehörigkeit in der belgischen Kolonialzeit unstrittig war, im Gefolge ethnischer Unruhen nach der Unabhängigkeit aber de facto vielen Angehörigen dieser Volksgruppe entzogen wurde. Die Gesetzgebung des Jahres $1971^{109}$ dehnte die Staatsangehörigkeit durch Zugehörigkeit bei Staatsgründung wieder erheblich aus und die Banyarwanda erwarben die zairische (kongolesische) Staatsangehörigkeit kollektiv zurück, wenn sie bei Eintritt des Kongo in die Unabhängigkeit am 30.6.1960 dort ansässig waren $^{110}$. Das wenig später nach heftigen Protesten ${ }^{111}$ in Kraft gesetzte Staatsangehörigkeitsgesetz von 1972 schaffte die Option einer doppelten Staatsangehörigkeit von mit Ausländern verheirateten Zairerinnen ab und billigte nur noch solchen Banyarwanda die Staatsangehörigkeit zu, welche vor dem $1.1 .1950^{112}$ in der Kivu-Region des Kongo ansässig waren und dies bis zur Unabhängigkeit blieben ${ }^{113}$.

Nachdem sich eine Gruppe von Banyarwanda mit einem Klagebrief an den Generalsekretär der Vereinten Nationen gewandt hatten, warf Präsident Mobutu am 26.3.1981 vor dem Zentralkomitee der Einheitspartei die seitdem vielfach zitierte Grundsatzfrage auf "Wer ist Zairer und wer ist dies nicht?". Die daraufhin wenige Monate später erfolgte vollständige Novellierung ${ }^{114}$ verschärfte die zuwanderungsfeindliche Tendenz des Vorgängergesetzes und schmälerte die Rechtsstellung Eingebürgerter durch die Unterscheidung zwischen einer "kleinen" und einer "großen" Staatsangehörigkeit drastisch. Noch gravierender formulierte das Gesetz hinsichtlich der Banyarwanda, dass Zairer (Kongolese) kraft originärer Zugehörigkeit nur war, wer einen Vorfahren hatte, welcher zu den bei Gründung des Freistaats Kongo am 1.8.1895 auf dessen Territorium ansässigen Stämmen gehörte und schloss damit sowohl Personen, deren Vorfahren später zugewandert waren als auch solche, die nicht in einem "Stamm" sozialisiert waren, mit dem Hinweis auf wirkliche und angebliche Miss-

107

Vereinigtes Königreich, Home Office, Immigration and Nationality Directorate, Democratic Republic of Congo - Country Report, London 2004, Ziff 5.4 ff.

108 Auch "kinyarwandi" bzw französisch "rwandophone", also "ruandisch-sprachig".

109

Verordnungs-Gesetz Nr 71-002 v 28.3.1971

110 Vgl. Tshibangu Tshiasu Kalala, Le droit zaïrois de la nationalité, RevJurZ 66/67 (1990/91), S. 7.

111 Vgl. Tshibangu Tshiasu Kalala (Fn. 110), S. 7.

112 Mit dieser Abgrenzung bleibt die Hauptgruppe der von der Kolonialverwaltung in der MasisiRegion angesiedelten Zuwanderer einbezogen.

113 Vgl. Tshibangu Tshiasu Kalala (Fn. 110), S. 7.

114 Gesetz Nr 81-002 v 29.6.1981 zur zairischen Staatsangehörigkeit. 
bräuche aus ${ }^{115}$. Diese "Feinheiten" sind insofern bedeutsam, als damit gezielt die Banyamulenge ${ }^{116}$ betroffen wurden, welche von keinem gemeinsamen Häuptling regiert wurden und deshalb als durch den Terminus "Stamm" nicht erfasst angesehen werden konnten ${ }^{117}$. Als während des zunehmenden Machtvakuums zu Ende der Mobutu-Ära der rechtlichen Ausgrenzung der Banyamulenge der physische Genozid zu folgen drohte, war dies der Auslöser für das militärische Eingreifen der Nachbarländer Ruanda sowie Burundi und Uganda und den folgenden Bürgerkrieg.

In der Friedensvereinbarung von Lusaka ${ }^{118}$ sowie dem die Transformation vorbereitenden Innerkongolesischen Dialog spiegelte sich die starke militärische Position der östlichen Nachbarn auch im Staatsangehörigkeitsrecht wieder, welches selbstverständlich einen zentralen Punkt der Verhandlungen bildete. Eckwerte wurden schließlich dahingehend festgelegt, dass es vorläufig (in der ersten Legislaturperiode der Transformation) beim Verbot der doppelten Staatsangehörigkeit bleiben sollte sowie die Einschränkungen des Staatsangehörigkeitserwerbs kraft Zugehörigkeit wieder weitgehend rückgängig gemacht werden sollten ${ }^{119}$. Diese Eckwerte wurden sodann in die Verfassung von $2003^{120}$ übernommen (Art 14 Verf). Verstärkt wurde dies dadurch, dass einer der vier Vizepräsidentenposten an einen Banyamulenge ging. Maßgeblicher Stichtag für die originäre Staatsangehörigkeit wurde wieder der bereits in der Gesetzgebung von 1971 zugrunde gelegte Unabhängigkeitstag und die Zugehörigkeit zu einer zu diesem Zeitpunkt ansässigen "ethnischen Gruppe" wurde als ausreichend angesehen. Anders als 1971/72 wurden die hauptsächlich betroffenen Bevölkerungsgruppen allerdings nicht ausdrücklich genannt, so dass für die Zukunft ein Rest an Unsicherheit blieb. Diese Position wurde durch die Gesetzgebung des Jahres $2004^{121}$ lediglich einfachgesetzlich umgesetzt (Art 4, 6 StAG); der Versuch, ein die Einzelheiten ausdrücklich regelndes Kapitel aufzunehmen, scheiterte ${ }^{122}$. Die Verfassung von 2005 schreibt den damit erreichten Rechtszustand fort (Art 10 Verf(2005)).

115 Kandolo M., Beaucoup de dirigeant congolais ont la double nationalité, Le Phare (Kinshasa) v 29.7.2004.

116 Vgl. Katunda/Matre/Mbuyi, Le manifeste de la paix, Kinshasa 2002, S. 3 ff.

117 Kandolo M., Beaucoup de dirigeants congolais ont la double nationalité, Le Phare (Kinshasa), 29.7.2004.

118 Katunda/Matre/Mbuyi (Fn. 118), S. 20.

119 Entschließung Nr DIC/CPR/03.

120 Übergangsverfassung in der Fassung v 4.4.2003, verö JO Sonderausgabe v. 5.4.2003, S. 3 ff.

121 Gesetz Nr 04-024 v 12.11.2004 über die kongolesische Staatsangehörigkeit, verö JO, Sonderausgabe v. 17.11.2004, iK 17.11.2004.

122

So schlug der federführende Ausschuss einen Sonderstatus für die Banyarwanda vor, vgl $G k$, Le Sénat rejette la nationalité par intégration, Le Phare (Kinshasa) v 9.9.2004. 


\section{Staatsorgane}

Als Staatsorgane werden nur - in dieser Reihenfolge - der Staatspräsident, das Parlament (welches im Vergleich mit der Verfassung von 2003 (Art 64) einen Platz höher rückt - aber den Realitäten entsprechend weiter hinter dem Staatspräsidenten rangiert), die Regierung (welche im Vergleich zur Verfassung von 2003 dem Parlament den Vortritt lassen muss) und die Gerichte bezeichnet (Art 68 Verf(2005)). Weitere durch die Verfassung vorgesehene Institutionen wie der Rechnungshof, die Unabhängige Wahlbehörde, die Hohe Behörde für Medien und Audivisuelles, der Oberste Richterrat, der Nationale Sicherheit oder der Ministerpräsident sind also keine Staatsorgane in diesem herausgehobenen Sinne. Auch der Verfassungsgerichthof wird im Unterschied zum Entwurf von 1998 (Art. 78) zumindest nicht ausdrücklich erwähnt. Anders als im Entwurf von 1998 wird zwischen diesen Zweigen auch kein Grundsatz der Gewaltenteilung postuliert, so dass die Aufzählung mehr die Absichten des Gesetzgebers erläutert als regelnden Charakter aufweist.

\section{Staatspräsident, Vizepräsidenten}

a) Ausgangslage

Abgesehen von der ersten Zeit nach Erlangen der staatlichen Unabhängigkeit ist der Kongo stets von einem übermächtigen, autokratisch herrschenden Staatspräsidenten regiert worden $^{123}$. Andererseits war gerade dieser Staatspräsident auch immer wieder Symbol und Identifikationskern eines heterogenen, vielfach in sich gespaltenen Landes. Die neue Verfassung steht nun vor der Aufgabe, die Schaffung von Demokratie mit Stabilität und Kohäsion der Teile des Gemeinwesens in Einklang zu bringen. Die künftige Rolle des Präsidenten war deshalb einer der wichtigsten Diskussionspunkte bei der Ausarbeitung der Verfassung.

\section{b) Wahlverfahren}

Die Wahl des Staatspräsidenten geschieht unmittelbar durch das Volk (Art 72 Verf(2005)). Sie hat 90 Tage vor Ablauf der Mandatszeit des gegenwärtigen Amtsinhabers stattzufinden (Art 73 Verf(2005)). Im ersten Wahlgang ist gewählt, wer die absolute Mehrheit der abgegebenen Stimmen erringt; erfüllt keiner der Bewerber dieses Kriterium, findet innerhalb von 14 Tage eine Stichwahl zwischen den beiden bestplatzierten Kandidaten statt (Art 71 Verf(2005)). Wählbar ist, wer gebürtiger Kongolese ist, das 30. Lebensjahr vollendet hat, im Vollbesitz seiner bürgerlichen Ehrenrechte steht sowie bei welchem kein wahlgesetzli- 
cher Ausschlussgrund greift (Art 72 Verf(2005)). 10 Tage nach Verkündung des amtlichen Wahlergebnisses tritt der neue Präsident sein Amt an (Art 74 (1) Verf(2005)).

\section{c) Wählbarkeit}

Im Laufe der Ausarbeitung wurde das Mindestalter der Kandidaten für das Amt des Staatspräsidenten besonders intensiv diskutiert ${ }^{124}$. Der Verfassungsentwurf des Senats, welcher das Mindestalter auf 45 Jahre hatte festlegen wollen, hätte damit zwar eine erneute Kandidatur des amtierenden Staatspräsidenten Kabila, welcher bei den Wahlen Mitte 2006 erst das 34. Lebensjahr vollendet haben wird, keineswegs ausgeschlossen, da ihm ebenso wie seinen Konkurrenten Bemba von der MLC und Ruberwa von der RCD eine Ausnahmeklausel die Bewerbung ermöglicht hätte, doch hätte dies die Legitimität der Kandidaturen beeinträchtigt. In der Diskussion wurde zugunsten einer Altersgrenze von 40 Jahren die bisherige Rechtslage im Kongo und in den meisten anderen afrikanischen Ländern angeführt, während für einen völligen Verzicht auf eine Altergrenze die notwendige Verjüngung in der kongolesischen Politik betont wurde ${ }^{125}$. Dieses wurde gegen heftige Widerstände auf 30 Jahre festgesetzt.

Ein offenkundiges Missbrauchspotential bietet der Ausschlussgrund des wahlgesetzlichen Bewerbungshindernisses; er sollte in seiner Anwendung deshalb auf die Beachtung der verfahrensmäßigen Bestimmungen beschränkt bleiben.

\section{d) Befugnisse und Aufgaben}

Der Präsident ist das Staatsoberhaupt (Art 69 Verf(2005)). Er hat nicht nur protokollarische Aufgaben, sondern verfügt entsprechend dem französischen Vorbild starke Kompetenzen in Außenpolitik, Verteidigung, Gesetzgebung, Justizwesen und Exekutive (insbes Art 91 Verf(2005)). Der Umfang dieser Befugnisse war während der Arbeiten zur Verfassungsgebung heftig umstritten ${ }^{126}$.

124

Lwazu di Tezo, La face cache du séminaire inter-institutionnel, Le Potentiel (Kinshasa) v 5.3.2005; Médard Muyaya, Assemblée nationale: l'avant-projet de Constitution renvoyé à la Commission Paj, Le Potentiel (Kinshasa) v 13.4.2005.

125

Médard Muyaya (Fn. 124).Assemblée nationale: l'avant-projet de Constitution renvoyé à la Commission Paj, Le Potentiel (Kinshasa) v 13.4.2005.

126

Fidèle Musangu, Azarias Ruberwa rejette la Constitution, Le Phare (Kinshasa) v. 26.4.2005; Les deputes préparent une dictature "constitutionelle", Le Potentiel (Kinshasa) v. 26.4.2005; Jean N'saka Wa N'saka, Constitution de la IIIème République: une bataille inutilement dure au Parlement, Le Phare (Kinshasa) v. 4.3.2005. 
Er vertritt die Nation nach außen (Art 69, 213 ff Verf(2005)), ernennt die kongolesischen Botschafter im Ausland (wofür er allerdings nach der neuen Verfassung der Gegenzeichnung durch den Premierminister bedarf, Art 81 Verf(2005)) und akkreditiert die ausländischen Botschafter im Kongo (Art 88 Verf(2005)). Ferner verhandelt und ratifiziert er internationale Vereinbarungen (Art 213 Verf(2005)); insbesondere im Falle von Eingriffen in die Gesetzgebung oder der Bindung von Haushaltsmitteln bedarf er hierfür allerdings einer gesetzlichen Grundlage (Art 214 Verf(2005)).

Militärisch ist er Oberkommandierender der Streitkräfte (Art 83 Verf(2005)) und ernennt deren Offiziere (wobei auch hier jetzt die Gegenzeichnung des Premierministers erforderlich ist, Art 81 Verf(2005)). Außerdem obliegt ihm die Kriegserklärung gegen fremde Staaten (Art 86, 143 Verf(2005)); er hat hierzu zuvor den Sicherheits- und Verteidigungsrat anzuhören und die Zustimmung beider Parlamentskammern einzuholen.

Im Justizwesen ist er auch weiter für die Ernennung von Richtern zuständig, allerdings obliegt das Vorschlagsrecht künftig einem ausschließlich mit Vertretern des Justizwesens besetzten Obersten Richterrat (Art 82 Verf(2005)). Ob das darüber hinaus auch hier eingeführte Erfordernis der Mitzeichnung durch den Premierminister die Unabhängigkeit der Justiz stärken oder deren Politisierung verfestigen wird, bleibt abzuwarten. Ferner behält der Präsident das Begnadigungsrecht (Art 87 Verf(2005)).

Im Bereich der Gesetzgebung hat der Präsident in den nicht der parlamentarischen Gesetzgebung vorbehaltenen Materien (Art 122 ff (Verf(2005)) ein eigenständiges Regelungsrecht, er bedarf allerdings künftig auch hier der Mitzeichnung des Premierministers (Art 79 (3), (4) Verf(2005)). Gegen vom Parlament beschlossene Gesetze hat er ein aufschiebendes Vetorecht (Art 137 Verf(2005)) und ist anderenfalls für deren Verkündung zuständig (Art 79 (2) Verf(2005)). Unter bestimmten Voraussetzungen kann er auch das Parlament auflösen (Art 148 Verf(2005)).

Vor allem hat der Präsident aber eine starke Stellung im Bereich der Exekutive. So ernennt der Präsident den Premierminister, welcher zwar zu einer parlamentarischen Mehrheit gehören muss, bei dessen Auswahl der Präsident aber große Freiheiten hat. Auch ist der Präsident und nicht der Premierminister derjenige, welcher die Kabinettssitzungen einberuft, die Tagesordnung festlegt und die Sitzungen leitet (Art $78 \mathrm{ff} \operatorname{Verf}(2005)$ ). Auf die Festlegung der Politik hat er maßgeblichen Einfluss (Art 91 (1) Verf(2005)). Für die Ernennung leitender Beamten sowie der Staatsbeauftragten in Unternehmen bedarf er künftig der Mitzeichnung durch den Premierminister (Art 81 (2) Verf(2005)). Die Provinzgouverneure sind zwar künftig direkt zu wählen, sie kommen aber weiter erst nach Ernennung durch den Staatspräsidenten in ihr Amt (Art 80, 198 Verf(2005)). Unklar ist, ob der Staatspräsident hierdurch über die Möglichkeit verfügt, nicht genehme Prätendenten trotz 
Wahl abzulehnen. Ferner ist er für die Verhängung des Ausnahme- und Belagerungszustandes zuständig (Art 85 ff, $144 \operatorname{Verf}(2005)$ ).

e) Amtsstellung

Die Amtsdauer des Staatspräsidenten beträgt 5 Jahre mit einmaliger Möglichkeit der Wiederwahl (Art 70 Verf(2005)); eine Verlängerung selbst im Wege einer Verfassungsänderung ist ausgeschlossen ((Art 220 (1) Verf(2005)). Nicht thematisiert wird die absehbare Frage, ob der derzeitige Amtsinhaber in Falle seiner Wahl im Jahr 2011 erneut kandidieren darf; da seine erste Amtsübernahme 2001 auf einer anderen verfassungsrechtlichen Grundlage und nicht durch Wahl erfolgte, dürften einer solchen Entwicklung aber keine verfassungsrechtlichen Hindernisse entgegenstehen.

Für den Staatspräsidenten gilt eine umfassende Inkompatibilität mit jedweder anderen beruflichen Tätigkeit einschließlich sämtlicher Wahl-, Verwaltungs- und Militärfunktionen sowie von Parteiämtern (Art 96 Verf(2005)). Auch ist ihm der unmittelbare oder mittelbare Erwerb von Staatseigentum untersagt (Art 98 Verf(2005)). Vor Amtsantritt und bei Ausscheiden hat er das gesamte Familienvermögen (also einschließlich dessen seiner Frau gemäß dem jeweiligen Güterstand sowie der vom Ehepaar unterhaltenen Kinder, auch wenn sie volljährig sind) offenzulegen (Art 99 Verf(2005)).

Im Falle einer dauerhaften Verhinderung des Staatspräsidenten führt der Senatspräsident ${ }^{127}$ die Amtsgeschäfte (Art 75 Verf(2005)). Die Amtsverhinderung ist in einem förmlichen Verfahren auf Antrag der Regierung durch das Verfassungsgericht auszusprechen; innerhalb einer Frist von 60 bis 90 Tagen sind dann Neuwahlen durchzuführen (Art 76 Verf(2005)).

Angesichts der allgemeinen Kritik an der vorherrschenden Straflosigkeit kam im Rahmen der Verfassungsdiskussion der Regelung der Durchführung von Strafverfahren gegen die obersten Amtsträger ein besonderer Stellenwert $\mathrm{zu}^{128}$. Im Ergebnis wurde von einer allgemeinen Immunitätsregelung abgesehen; lediglich Straftaten, welche nicht in Ausübung, sondern lediglich bei Gelegenheit der Amtsführung verübt wurden, können erst nach Ausscheiden aus dem Amt verfolgt werden. Zusätzlich wurde ein wenig transparenter, inhomo-

127 In der Verfassungsdiskussion war zunächst strittig, ob nicht dem Sprecher des Abgeordnetenhauses der Vorzug gegeben werden sollte; in der Diskussion, vgl. Médard Meyay, Constitution: démarrage des travaux de la commission politique, administrative et judiciaire, Le Potentiel (Kinshasa) v. 15.4.2005.

128 Angelo Mobateli, Appel des évêques catholiques aux politiques congolais: des projets des societé en phase avec les desiderata du people, Le Potentiel (Kinshasa) v 7.3.2006. 
gener und kaum operationaler Katalog von Straftatbeständen festgelegt (Art 164 ff Verf(2005)) ${ }^{129}$. Dazu gehören neben in Ausübung oder bei Gelegenheit der Amtsführung begangenen Straftaten des gemeinen Recht der Hochverrat (welcher als bewusste Verfassungsverletzung, schwerwiegende Verletzung der Menschenrechte oder die Abtretung von Staatsgebiet definiert wird) die Ehrverletzung (Verstoß gegen die guten Sitten, Verschwendung staatlicher Mittel, Korruption oder unerlaubte Bereicherung), die Vornahme verbotener Insider-Geschäfte sowie die Missachtung des Parlaments (Nichtbeantwortung parlamentarischer Anfragen innerhalb einer Frist von 30 Tagen). In solchen Fällen fungiert das Verfassungsgericht als Strafgericht für den Präsidenten und Premierminister (Art 164 VerfG(2005)). Zur Einleitung eines solchen Verfahrens ist ein Beschluss von 2/3 der Mitglieder des in einer gemeinsamen Sitzung vereinten Kongresses erforderlich (Art 166 Verf(2005)). Kommt es zur Verurteilung, schließt diese die Amtsenthebung ein (Art 167 (1) $\operatorname{Verf}(2005))$.

\section{f) Vizepräsidenten}

Das System von 4 den unterschiedlichen politischen Familien angehörigen Vizepräsidenten als Zwischen- und Nebeninstanz zu Staatspräsident und Regierung hat sich nach allgemeiner Überzeugung nicht bewährt ${ }^{130}$. So fehlte es jedem der 4 Vizepräsidenten sowohl an eigenen Entscheidungskompetenzen als an einem verwaltungsmäßigen Unterbau, so dass es keinem von ihnen gelang, sich als eine anerkannte staatliche Instanz zu etablieren. Die neue Verfassung verzichtet konsequenterweise auf diese Institution.

\section{Ministerpräsident, Regierung}

\section{a) Entwicklung}

Das in der Verfassung des Brüsseler Runden Tisches unzureichend geregelte Verhältnis zwischen Staatspräsidenten und Ministerpräsidenten erwies sich schon unmittelbar nach Unabhängigkeit des Kongo als problematisch und es kam zu einem heftigen Machtkampf, welchen schließlich der zeitweilige Ministerpräsident und spätere Staatschef Mobutu für sich entscheiden konnte. In der Endphase der Mobutu-Ära erschien das Amt des Minister- 
präsidenten als eine Möglichkeit, die bisherige politische Opposition zumindest optisch in die Regierungsverantwortung mit einzubeziehen ${ }^{131}$.

\section{b) Verfassungsrechtliche Regelung}

So war während des Prozesses der Verfassungsgebung die Verteilung der Machtbefugnisse zwischen Staatspräsidenten und Ministerpräsidenten heftig umstritten. Im Ergebnis wurde ein semi-präsidentielles System beibehalten, in welchem die Stellung des Premierministers etwas stärker als im Entwurf des Senats vorgesehen ausgestaltet wurde. Nach der letztlich verabschiedeten Konzeption liegt das Schwergewicht der politischen Befugnisse eindeutig beim Staatspräsidenten, während der Ministerpräsident weitgehend von diesem abhängig bleibt (vgl insbes Art 91 (1) Verf(2005)) ${ }^{132}$. Zwar leitet der Ministerpräsident grundsätzlich die Regierung und die politische Verwaltung, ist dem Parlament verantwortlich (Art 91 (5) Verf(2005) und muss aus den Reihen der parlamentarischen ${ }^{133}$ Mehrheit $^{134}$ stammen (Art 78 Verf(2005)) sowie vor der Übernahme seines Amtes eine mehrheitliche Billigung seines Regierungsprogramm durch das Parlament erreichen (Art 90 (4), (5) Verf(2005)). Seine Auswahl liegt jedoch letztlich in den Händen des Staatspräsidenten, zumal der Begriff der "politischen Mehrheit" vage bleibt und offen lässt, wie im Falle einer relativen Mehrheit oder unterschiedlichen Stimmenverhältnissen in den beiden Kammern zu verfahren ist ${ }^{135}$. Die Kabinettssitzungen werden nicht von ihm, sondern vom Staatspräsidenten einberufen und geleitet (Art 79 Verf(2005)). Ebenso bleiben die wichtigsten Personalentscheidungen in den Händen des Staatspräsidenten (Art 81 Verf(2005)); das neu eingefügtes Recht zur vorherigen Mitzeichnung bestimmter Entscheidungen (insbes Art 79, 81, $82 \operatorname{Verf(2005))}$ verschaffen dem Ministerpräsidenten zwar Mitentscheidungsmöglichkeiten, aber kaum eigenständigen Gestaltungsspielraum.

Die Regierung muss regional und ethnisch ausgewogen sein (90 (3) Verf(2005)), also die unterschiedlichen ehemaligen Bürgerkriegsfraktionen integrieren. Sie setzt sich aus dem Premierminister, den Ministern und Vizeministern sowie ggf. einem stellvertretenden Premierminister, Staatsministern und Juniorministern (nach dem französischen Vorbild dürften

Vereinigtes Königreich, Home Office, Immigration and Nationality Directorate, Democratic Republic of Congo - Country Report, London 2004, Ziff 5.9.

132 Auguste Mampuya Kanunk-a-Thsiabo, Le projet de Constitution n'est pas encore prêt pour être soumis à un referendum, Le Phare (Kinshasa) v. 31.8.2005; AM, Projet de Constitution: Copie à refaire, Le Potentiel (Kinshasa) v 30.5.2005.

133

134

Gemeint ist offenbar die Mehrheit im Abgeordnetenhaus, vgl. AM (Fn.132).

135

Gemeint ist anscheinend die Mehrheit im Abgeordnetenhaus, vgl. AM (Fn.132).

Auguste Mampuya Kanunk-a-Thsiabo (Fn. 132). 
darunter Minister mit beschränktem Aufgabengebiet unter der Gesamtverantwortung eines Titularministers gemeint sein) zusammen (Art 90 Verf(2005)).

Die Inkompatibilität der Regierungsämter entspricht den Regeln für den Staatspräsidenten mit der Abweichung, dass landwirtschaftliche, handwerkliche, kulturelle, Unterrichts- und Forschungstätigkeiten erlaubt sind. Gleichfalls gelten die strengen Transparenzregeln für die Offenlegung des Familienvermögens. Auch die Regeln für die Immunität der Regierungsmitglieder richten sich nach den für den Präsidenten geltenden Bestimmungen; Strafverfahren gegen den Premierminister sind ebenfalls vor dem Verfassungsgericht durchzuführen. Über Straftaten anderer Regierungsmitglieder entscheidet dagegen der Kassationsgerichtshof (Art 153 (3) Verf(2005)) ${ }^{136}$. Zur Einleitung eines solchen Verfahrens ist ein Beschluss der Mehrheit der Mitglieder des in einer gemeinsamen Sitzung vereinten Kongresses erforderlich (Art 166 Verf(2005)). Eine solche Anklageerhebung führt automatisch zur Abberufung des betreffenden Regierungsmitglieds.

Als weitere Gründe für das Ausscheiden aus dem Amt werden nur der Amtsverlust aufgrund Rücktritts, des Verlusts einer parlamentarischen Vertrauensabstimmung sowie eines parlamentarischen Misstrauensvotums geregelt; ob der Staatspräsident ihn in Umkehrung seines Rechtes zu seiner Ernennung auch abberufen darf, ist nicht ausdrücklich geklärt, hier bleibt die weitere Verfassungspraxis abzuwarten. Die Regierung kann die Vertrauensfrage in Zusammenhang mit ihrem Programm, einer politischen Frage oder einem Rechtstext stellen (Art 146 Verf(2005)). Ein Misstrauensantrag gegen die gesamte Regierung kann von einem Viertel der Abgeordneten eingebracht werden, ein solcher gegen einen einzelnen Minister von einem Sechstel der Abgeordneten. Er ist während einer parlamentarischen Sitzungsperiode von 3 Monaten jeweils nur ein einziges Mal möglich. Wird die Vertrauensfrage in Zusammenhang mit einem Rechtstext gestellt und kommt keine Mehrheit gegen die Regierung zustande, ist dieser Text damit angenommen, anderenfalls gilt die Regierung als zurückgetreten (Art 146, $147 \operatorname{Verf}(2005))$.

\section{3. Öffentlicher Dienst}

\section{a) Entwicklung}

Die Öffentliche Verwaltung gehört zu den wesentlichen Schwachpunkten des kongolesischen Staatswesens ${ }^{137}$. Physisch existieren zwar im gesamten Land Verwaltungsstrukturen,

\footnotetext{
$136 A M$ (Fn. 132).
}

137 Bertelsmann Transformation Index 2003, Länderbericht Demokratische Republik Kongo, Gütersloh 2003, S 5 . 
welche jedoch aufgrund von Ressourcenmangel, Korruption und interne Spaltung jedoch nahezu funktionsuntüchtig sind. Die Regierung kontrolliert nur einen Teil des Staatsgebiets, während in den übrigen Gebieten weiter die Willkür von Milizen, ethnischer Gruppen und örtlicher Machthaber herrscht ${ }^{138}$. Ein großer Teil der ländlichen Bevölkerung hat keinen Zugang zu staatlichen Infrastrukturen. Öffentliche Bedienstete sind oft schlecht ausgebildet und gering oder oft auch über Monate hinweg gar nicht bezahlt ${ }^{139}$. Korruption und Vetternwirtschaft sind an der Tagesordnung. Die Maßnahmen aufgrund der Verfassung von 2003 haben kaum zur Verbesserung der Verwaltungsführung beigetragen. Dies gilt auch für die von der Verfassung von 2003 zur Sicherung guter Verwaltungsführung, Transparenz und Beachtung ethischer Grundsätze eingerichtete Kommission für Ethik und Korruptionsbekämpfung ${ }^{140}$.

\section{b) Verfassungsrechtliche Regelung}

Die Verwaltung muss unpolitisch und unparteilich sein. Sie darf nicht zu persönlichen oder parteilichen Zwecken eingesetzt werden (Art 193 (1) Verf(2005)). Die Korruptionsbekämpfungskommission wird angesichts ihrer Wirkungslosigkeit in der neuen Verfassung nicht mehr erwähnt. Als wirksamer könnte sich mittel- und langfristig die Einrichtung einer Verwaltungsgerichtsbarkeit und die Stärkung des Rechnungshofs erweisen.

\section{Parlament}

a) Entwicklung

Nach dem raschen Scheitern des Parlamentarismus der ersten Verfassungen nach der Unabhängigkeit führte das Parlament in der Mobutu-Ära ein Schattendasein. Einen ersten Ansatz für eine Wiederbelebung des Parlamentarismus bildete die im Juli 1991 einberufene Nationalkonferenz, bis 1994 ein - weitgehend machtloses - Übergangsparlament aus dem von der Nationalkonferenz eingesetzten Hohen Rat und früheren Nationalversammlung gebildete wurde. Nachdem freie Wahlen zunächst nicht zustande kamen, wurde im August 2000 ein Übergangsparlament mit von den Bürgerkriegsfraktionen benannten Mitgliedern eingesetzt. Diesem Übergangsparlament fiel es von Anfang an schwer, sich der Bevölke-

Tshiala David, Kasaï Oriental: mise en garde contre la mauvaise gestion de la chose politique, Le Potentiel (Kinshasa) v 6.3.2006; Sylvain Kapuya, Katanga: des agents de L'ANR et la police sèment la désolation à Malemba Nkulu, Le Potentiel (Kinshasa) v 27.2.2006.

139 Tshiala David, Nord-Kivu: la ville de Goma attend l'arrivée du chef de l'Etat, Le Potentiel (Kinshasa) v. 4.3.2006.

140

Langana et al.(Fn. 51), S. 7. 
rung als Autor einer modernen Gesetzgebung zu präsentieren, vielmehr überwog der Eindruck schleppender und begrenzt sachorientierter Gesetzgebungsarbeit ${ }^{141}$. Nach Jahrzehnten der Gleichschaltung und Unterdrückung der Demokratie hat das im Juli 2006 neu zu wählende Parlament erstmalig die Chance auf eine maßgebliche Gestaltung der Politik des Landes.

\section{b) Organisation des Kongresses}

Der Kongress wird wiederum aus zwei Kammern bestehen, nämlich dem Abgeordnetenhaus und dem Senat (Art 100 (1) Verf(2005)). Er beschließt die Gesetze und kontrolliert die Regierung, die öffentlichen Betrieben und sonstigen öffentlichen Einrichtungen (Art 100 (2) Verf(2005)).

\section{c) Abgeordnetenhaus}

Das Abgeordnetenhaus hat die vorrangige Rolle bei der Gesetzgebung. Die Abgeordneten vertreten die Nation als Ganzes (Art 101 (4) Verf(2005)); das für das Übergangsparlament geltende imperative Mandat $^{142}$ ist künftig ausgeschlossen (Art 101 (5) Verf(2005)).

Die Wählbarkeit als Abgeordneter setzt kongolesische Staatsangehörigkeit voraus (welche anders als im Falle des Staatspräsidenten auch nachträglich erworben sein darf), die Vollendung des 25. Lebensjahres, den Vollbesitz der bürgerlichen Ehrenrechte sowie das Fehlen eines wahlgesetzlichen Ausschlussgrundes voraus (Art 102 Verf(2005)). Die Mandatszeit beträgt 5 Jahre, eine beliebige Wiederwahl ist zulässig (Art 103 (Verf(2005)).

Bei fortgesetzter Krise zwischen Regierung kann der Staatspräsident das Abgeordnetenhaus (nicht den Senat) auflösen. Der Staatspräsident verfügt damit über ein wichtiges Instrument der Politikgestaltung.

\section{d) Senat}

Die Senatoren werden in indirekter Wahl von den Provinzparlamenten entsandt (Art 104 (4) Verf(2005)). Zusätzlich sind die ehemaligen Staatspräsidenten geborene Mitglieder des Senats auf Lebenszeit (Art 104 (7) Verf(2005)); der Senatsentwurf (Art 217) hatte dagegen auch die bisherigen 4 Vizepräsidenten und die Vorsitzenden der beiden Kammern als geborene Senatoren vorgesehen. Damit wären allerdings die frühere Opposition gegen den

141 Langana et al.(Fn. 51), S. 5; Vereinigtes Königreich, Home Office, Immigration and Nationality 142 Directorate, Democratic Republic of Congo - Country Report, London 2004, Ziff 5.8.

s. oben 2 e bb. 
Staatspräsidenten zahlenmäßig bevorzugt worden, auch hatte sich der Präsident des Abgeordnetenhauses mit der ihn entsendenden Fraktion überworfen, hatte der als Vertreter der Zivilgesellschaft berufene Vizepräsident dort von Anfang keinen Rückhalt und es waren der dritte Vizepräsident als Angehöriger der ruandaphonen Minderheit der Banyamulenge sowie der vierte Vizepräsident als aus dem engeren Umfeld Mobutus kommend politisch schwer vermittelbar. Die Senatoren vertreten ihre jeweilige Provinz, haben aber ein nationales Mandat (Art 104 (2) Verf(2005)). Auch Senatoren gegenüber ist ein imperatives Mandat ausgeschlossen (Art 104 (3) Verf(2005)). Die Wählbarkeitsvoraussetzungen sind die gleichen wie bei den Abgeordneten, nur dass ein Mindestalter von 30 Jahren gefordert wird (Art 166 Verf(2005)).

\section{e) Persönliche Stellung der Parlamentarier}

Die Mandatsdauer beträgt jeweils 5 Jahre (Art 105 Verf(2005)). Das Mandat endet vorzeitig insbesondere im Falle des Rücktritts, der ungerechtfertigten und ungenehmigten Abwesenheit von mehr als einem Viertel der Sitzungen einer 3-monatigen Sitzungsperiode, des Vorliegens eines wahlgesetzlichen Ausschlussgrundes oder der Annahme einer nicht kompatiblen anderweitigen Funktion (Art 110 Verf(2005)).

Die Inkompatibilität ist für Abgeordnete und Senatoren identisch geregelt. In beiden Fällen ist eine Kumulation mit Ämtern in der Regierung, den Streitkräften, der Justiz, der Zentraloder Lokalverwaltung oder irgendeinem anderen Wahlamt ausgeschlossen (Art 108 (Verf(2005)). Problematisch erscheint der dieser Bestimmung offenbar zugrundeliegende Automatismus, wonach anscheinend keine Wahlmöglichkeit zwischen den inkompatiblen Ämtern zu bestehen scheint, sondern zwingend das parlamentarische Mandat verloren geht (Art 110 (Verf(2005)). Parlamentarier genießen ferner verfassungsrechtliche Immunität; ein Parlamentarier kann strafrechtlich nur verfolgt werden, wenn er auf frischer Tat ertappt wird oder seine Parlamentskammer dem zustimmt (bzw. außerhalb der Sitzungsperioden der jeweilige Vorstand der Kammer, Art 107 Verf(2005)).

\section{f) Geschäftsordnung}

Jede der beiden Parlamentskammern hat sich eine Geschäftsordnung zu geben, welche insbesondere das Ausschusswesen und das Abstimmungsverfahren regelt (Art 112 Verf(2005)). Jede Kammer hat sich einen 7-köpfigen Vorstand zu geben (Art 111 Verf(2005)). Jedes Jahr finden zwei jeweils 3-monatige Sitzungsperioden vom 15.3. bis 15.6. bzw 15.9. bis 15.12. statt (Art 115 Verf(2005)).Die Regierung kann jederzeit verlangen, dass ein Gegenstand vorrangig auf die Tagesordnung des Parlaments gesetzt wird (Art 111 Verf(2005)). Das Quorum für eine Beschlussfassung liegt bei der Mehrheit der gesetzlichen Mandate (Art 118 Verf(2005)). 
Auf eine ausdrückliche Regelung des Status der Opposition wurde bereits im Vorentwurf des Senats verzichtet, da dies der freien Entscheidung der Opposition überlassen werden sollte $^{143}$. Gleichwohl soll nach dem vom Abgeordnetenhaus verabschiedeten Text zu dieser Thematik ein Organgesetz ergehen (Art 8 (2) Verf(2005)). Im übrigen dürfen die Rechte der Opposition nur durch die für alle Parteien und politischen Aktivitäten geltenden gesetzlichen und verfassungsmäßigen Grenzen eingeschränkt werden.

\section{Gesetzgebung}

Im Rahmen der Diskussion des Entwurfs im Abgeordnetenhaus gab es zwar Versuche, dem Parlament entsprechend dem angelsächsischen Modell ein umfassendes Gesetzgebungsrecht zu verschaffen, im Ergebnis sprach sich die Mehrheit jedoch für das französische Modell eines enumerativen Katalogs von der Gesetzgebung unterworfenen Materien aus ${ }^{144}$. $\mathrm{Zu}$ dem nicht sehr umfangreichen Katalog der der Gesetzgebung vorbehaltenen Materien zählen u.a. die Gebiete des Wahlrechts, der Staatsangehörigkeit, des Familienstandes, des Familien- und Erbrechts, des Handels, des Rechtes der Arbeit und der sozialen Sicherheit, des Steuerrechts, des Beamtenrechts und des Strafrechts (Art 122 Verf(2005)) sowie die Grundsätze ua der Materien der Lokalverwaltung, der Unternehmensgründung, des Bodenund Rohstoffrechts, des Streikrechts, der Forschung, der Kultur und der Kunst (Art 123 Verf(2005)). Alle anderen Materien sind durch Verordnung des Staatspräsidenten (Art 79 (3), 128 Verf(2005)) bzw durch Erlass der Regierung (Art 92 (2) Verf(2005)) zu regeln. Bereits ergangenen Gesetze können allerdings nur nach verfassungsgerichtlicher Prüfung geändert werden. Für begrenzte Dauer und für ein begrenztes Themengebiet ist es auch möglich, die Gesetzgebungskompetenz zur Ausübung der Gesetzgebungstätigkeit zu ermächtigen. Die Regierung entscheidet dann durch Verordnungsgesetze, welche ihre Wirkung verlieren, wenn sie nicht vor Fristablauf durch ein Parlamentsgesetz ratifiziert werden (Art 129 Verf(2005)).

Die Verfassung sieht ferner die Regelung bestimmter Materien durch sog. Organgesetze vor, wodurch besondere Ansprüche an den Verfahrensgang zu stellen sind; insbesondere hat das Verfassungsgericht die Verfassungskonformität bereits vor der Verkündung zu prüfen (Art 124 Verf(2005)); Änderungen im Eilverfahren sind ausgeschlossen (Art 125 (2) Verf(2005)). In diese Fallgruppe gehören etwa das Richtergesetz und das Gesetz über das Oppositionsstatut. 
Das Initiativrecht für das Einbringen von Gesetzesentwürfen steht sowohl der Regierung als auch jedem einzelnen Parlamentarier aus einer der beiden Kammern zu; die Regierung kann jederzeit Änderungen zu Parlamentsentwürfen vorlegen (Art 130 Verf(2005)).

Kommt es bei einem Gesetzesentwurf zu keiner Einigung der beiden Kammern, ist der mit einer gleichgroßen Zahl von Parlamentarien aus dem Abgeordnetenhaus und dem Senat besetzte Vermittlungsausschuss einzuschalten (Art 113, 135 Verf(2005)). Kommt es auch dort zu keiner Einigung, entscheidet jedoch das Abgeordnetenhaus abschließend. Es kann dann seine letzte eigene Fassung, eine solche aus dem Vermittlungsausschuss oder eine Fassung unter Einschluss von Änderungen des Senats zugrunde legen.

6 Tage nach der Annahme ist der Gesetzestext dem Präsidenten zur Verkündung zuzuleiten (Art 136 Verf(2005)). Letzter verfügt über ein aufschiebendes Vetorecht, welches jedoch mit der absoluten Mehrheit der Mitglieder von Senat und Abgeordnetenhaus überstimmt werden kann (Art 137 Verf(2005)); das im Senatsentwurf ähnlich wie in der Übergangsverfassung (Art 130 (2)) vorgesehene Erfordernis einer 2/3-Mehrheit wurde also deutlich abgemildert. Andernfalls hat er das Gesetz innerhalb von 15 Tagen zu verkünden. Tut er dies nicht, gilt das Gesetz gleichwohl von Rechts wegen als verkündet (Art 140 Verf(2005)). Damit wird eine Wiederholung des Vorgehens des früheren Präsidenten Mobutus verhindert, welcher sich durch das willkürliche Unterlassen der Verkündung ein unbegrenztes faktisches Vetorecht gesichert hatte. Vor Verkündung können der Präsident, die Regierung oder eine Gruppe von Parlamentariern in einer Stärke von mindestens 1/10 der Mitglieder der jeweiligen Kammer eine vorherige verfassungsgerichtliche Überprüfung verlangen. Wenn gesetzlich nichts anderes vorgesehen ist, treten Gesetze 30 Tage nach ihrer Verkündung in Kraft (Art 142 Verf(2005)).

\section{Parteien}

a) Entwicklung

Bei den Regionalwahlen 1957 wurden noch unter belgischer Kolonialherrschaft erstmals politische Parteien zugelassen ${ }^{145}$. Für die Wähler war dieses Angebot allerdings wenig transparent, so dass bei Eintritt in die Unabhängigkeit eine kaum überschaubare Vielfalt von Parteien um die Macht kämpfte. Nachdem Mobutu seine Macht konsolidiert hatte, schaffte er diese Vielfalt zugunsten einer Einheitspartei nach sozialistischen Vorbildern ab. Unruhen führten 1990 dazu, dass Präsident Mobutu erneut den Übergang zu einem Mehrparteiensystem ausrief. Im November wurden Oppositionsparteien zugelassen. Am 
26.5.1997 verbot Präsident Kabila erneut alle politischen Parteien; im Mai 2001 wurde die politische Aktivität wiederum freigegeben.

Heute gibt es wieder zahlreiche Parteien ${ }^{146}$; bereits wenige Tage nach Inkrafttreten des neuen Wahlgesetzes hatten sich 270 Parteien beim Innenministerium registrieren lassen ${ }^{147}$. Allerdings sind die meisten von lediglich lokaler Bedeutung und das Vehikel politisch ambitionierter Einzelpersönlichkeiten ${ }^{148}$. Derzeit kann lediglich die UDPS ${ }^{149}$ des Oppositionsführers und zeitweiligen Ministerpräsidenten von Ex-Diktator Mobutu Etienne Tshisekedi überregional eine breitere Unterstützung für sich in Anspruch nehmen; sie hat eine Beteiligung an den bevorstehenden Wahlen jedoch abgelehnt.

\section{b) Verfassungsrechtliche Regelung}

Die Mitwirkung der politischen Parteien am politischen Leben des Landes ist garantiert (Art 6 Verf(2005)). Sie müssen die Grundsätze einer pluralistischen Demokratie, der staatlichen Einheit und der nationalen Souveränität achten. Sie können aufgrund gesetzlicher Regelungen Staatszuschüsse erhalten. Die Gründung einer Einheitspartei ist ausdrücklich untersagt (Art 7 Verf(2005)).

\section{Wahlen}

\section{a) Entwicklung}

Die bisherige Geschichte des Kongo hat freie und faire Wahlen verhindert. Der gegenwärtige Transformationsprozess bietet nun erstmalig die Chance einer demokratischen Besetzung der wesentlichen staatlichen Funktionen. Die aufgrund eines Gesetzes von $2004^{150}$ eingerichtete Unabhängige Wahlkommission ist zuständig für die Organisation und Durchführung von Referenden und Wahlen auf allen Ebenen. Sie ist durch Klientilismus und Tribalismus gekennzeichnet ${ }^{151}$. Hinzu kommt ein geringes Maß an fachlicher Kompe-

\footnotetext{
146 Vereinigtes Königreich, Home Office (Fn. 141), Ziff. 5.12 ff.

147 DRC: Registration of Presidential, Legislative Candidates Begins, VN-IRIN v 10.3.2006.

148 Vereinigtes Königreich, Home Office (Fn. 141), Ziff 5.13.

149 Union für Demokratie und sozialen Fortschritt (Union pour la démocracie et le progrès social).

${ }^{150}$ Gesetz Nr 04/009 v 5.6.2004 über die Organisation, Zuständigkeit und Tätigkeitsweise der Unabhängigen Wahlkommission.

151 Langana et al.(Fn. 51), S. 1, 6.
} 
tenz ${ }^{152}$. Gleichwohl hat sie das Verfassungsreferendum im Wesentlichen erfolgreich durchführen können. Für den weiteren Transformationsprozess kommt ihr auch künftig eine Schlüsselstellung zu. Als Grundlage der voraussichtlich am 30.7.2006 stattfindenden Präsidenten- und Parlamentswahlen hat das Parlament am 22.2.2006 ein Wahlgesetz ${ }^{153}$ verabschiedet. Danach findet die Wahl in einem einzigen Wahlgang mit zwei je nach Größe des Wahlkreises differierenden Wahlverfahren statt. Daran schließt sich die in zwei Runden durchzuführende Präsidentschaftswahl an. Das Wahlverfahren für das Präsidentenamt wurde im Laufe der Ausarbeitung der Verfassung heftig diskutiert; die Kritiker einer direkten Wahl, welche eine zu große Stärkung des Präsidenten gegenüber den anderen Institutionen befürchteten, konnten sich dabei nicht durchsetzen ${ }^{154}$. Präsidentschaftskandidaten haben gemäß dem Wahlgesetz eine nicht rückerstattbare Kaution von 50.000 US-\$ zu leisten ${ }^{155}$. Für beide Ämter war zunächst ein schleppender Eingang von Bewerbungen zu verzeichnen, zumal die Pflicht zur Leistung einer Kaution in dem armen Land eine hohe Hürde darstellte. Nach einer 10-tägigen Fristverlängerung kam ein großes Bewerberfeld zustande $^{156}$; insgesamt hatten bis zum Ablauf der Registrierung 73 Kandidaten ihre Bewerbung für das Präsidentenamt abgegeben. Von diesen wurden nach Prüfung der wahlgesetzlichen Voraussetzungen 33 zugelassen, darunter 4 Frauen ${ }^{157}$. Für die 500 Abgeordnetensitze kandidieren 9.362 Bewerber ${ }^{158}$. Das weitere Ziel, auch dem wichtigsten Oppositionellen Etienne Tshisekedi eine Brücke zum Einreichen einer Bewerbung zu bauen, konnte dagegen nicht erreicht werden ${ }^{159}$. Nachdem eine erste Frist zur Registrierung von Kandidaten eine unzureichende Zahl von Kandidaten erbracht hatte, musste noch einmal eine 10tägige Verlängerung gewährt werden ${ }^{160}$.

152

153

Langana et al.(Fn. 51), S. 7.

Gesetz Nr 006/06 v. 9.3.2006 über die Organisation der Präsidenten-, Parlaments-, Provinz-, Stadt- und Gemeindewahlen; Erlass Nr 004/CEI/BUR/06 v 9.3.2006; Médard Muyaya, Parlament: les deputés et sénateurs ont adopté la loi électorale, Le Potentiel (Kinshasa) v. 23.2.2006.

Fidèle Musangu, Azarias Ruberwa rejette la Constitution, Le Phare (Kinshasa) v. 26.4.2005; Les deputes préparent une dictature "constitutionelle", Le Potentiel (Kinshasa) v. 26.4.2005..

Der Sinn dieser umstrittenen Bestimmung liegt insbesondere darin, das Feld von vornherein auf solche Bewerber zu beschränken, welche über ausreichende Ressourcen für einen landesweiten Wahlkampf verfügen.

N.N:, La CEI prolonge le dépot des candidatures jusqu'au 2 avril, Le Potentiel (Kinshasa) v. 24.3.2006.

157 VN-IRIN v. 1.5.2005.

158

Calendrier électoral: incertitudes, Le Potentiel (Kinshasa) v. 5.4.2006.

159 P. Mbelu, Processus electoral, respect des texts et deficit de la pensée, Le Potentiel (Kinshasa) v. 28.3.2006; N.N (Fn. 156); Candidates Registered, Election Date Undecided, UN-IRIN v. 5.4.2006.

160

N.N. (Fn. 156); J.P Mbelu, Processus électoral, respect des texts et deficit de la pensée, Le Potentiel (Kinshasa) v. 24.3.2006. 
Die Verfassung bestätigt die Unabhängige Wahlkommission in ihrem Amt (Art 211 Verf (2005)) und schreibt vor, dass Wahlen allgemein, gleich und geheim sein müssen (Art 5 (4) Verf(2005)). Wahlberechtigt ist, wer volljährig und im Vollbesitz seiner bürgerlichen Ehrenrechte ist (Art 5 (5) Verf(2005)).

\section{Justizwesen \\ a) Entwicklung}

Das Fehlen eines verlässlichen Justizwesens gilt als eines der Haupthindernisse für den Wiederaufbau des Landes ${ }^{161}$. Die Schaffung eines Rechtsstaats kann dabei auf keinen gewachsenen Traditionen aufbauen, sondern ist in der Geschichte des Landes ein Novum. Sie wird nur mit Unterstützung ausländischer Geber und als langfristige Aufgabe Erfolg haben können ${ }^{162}$. Zwar ist die Justiz nominell unabhängig. Sie steht tatsächlich aber unter Kontrolle von Präsident und Verwaltung ${ }^{163}$ und wird als hochgradig korrupt beschrieben ${ }^{164}$. Auch die ethnisch ungleichgewichtige Besetzung beeinträchtigt die Akzeptanz des Justizwesens $^{165}$. Die rund 2.000 Richter $^{166}$ des Landes arbeiten zudem unter prekären materiellen Bedingungen ${ }^{167}$. Hinzu kommt, dass die im wesentlichen aus der Mobutu-Ära

$J r t$, Insécurité à Kinshasa: le seuil de l'intolérable, Le Potentiel (Kinshasa) v. 9.5.2006 Ziff C 2.4.

162 Vereinigtes Königreich, Home Office (Fn. 141), Ziff 5.15 ff.

163

Tshiambi,La RDC a besoin d'une justice réellement indépendante, Le Potentiel (Kinshasa) v. 12.1.2006.

164 So auch der seriöse Bertelsmann Transformation Index 2003, Länderbericht Demokratische Republik Kongo, Gütersloh 2003, S6; International Commission of Jurists, Attacks on Justice, 11. Aufl.. Genf, 2002, S 117 ff. ; Vereinigtes Königreich, Home Office (Fn. 141), Ziff 5.16; Zwischen 1998 und 2003 wurden 375 Richter wegen Korruptionsvorwürfen aus dem Amt entfernt; örtliche Menschenrechtsgruppen sahen darin allerdings einen Eingriff in die Unabhängigkeit der Justiz, IRIN v. 30.10.2003; J-Alian Kabongo, Après les paramédicaux et les enseigants, les magistrats de Kinshasa en grève, Le Phare (Kinshasa) v 22.2.2005.

166

Langana et al.(Fn. 51),: un bilan globalement négatif, Le Phare (Kinshasa) v 15.2.2005, S 7.

Im Zuge der Transformation wurde die Zahl der Richter insbesondere zugunsten unterversorgter, abgelegener Gebiete um rd. $15 \%$ aufgestockt, vgl. Kayembe, Justice: les arrêtés du ministre sèment la doute, Le Potentiel (Kinshasa) v 12.6.2006.

167

Im Jahr 2003 lag das Gehaltsniveau bei umgerechnet 10-20 USD pro Monat und es gab mehrmonatige Rückstände bei den Gehaltszahlungen; der bauliche Zustand und die technische Ausstattung der Gerichte ist unzulänglich, vgl IRIN v. 30.10.2003; Langana et al.(Fn. 51),: un bilan globalement négatif, Le Phare (Kinshasa) v 15.2.2005, S 7 spricht von "Clochardisierung". 
stammenden gesetzlichen Grundlagen unzureichend sind und die staatliche Beeinflussung erleichtern $^{168}$.

Weit über die Hälfte der Streitigkeiten machen Auseinandersetzungen über Bodenflächen aus, welche insbesondere im ehemals wohlhabenden, heute aber übersiedelten Osten des Landes knapp sind ${ }^{169}$. Die unterste Instanz bilden die Friedensgerichte (Tribuneaux de Paix), welche ua. für alle Streitigkeiten über Erb- und Familienrecht, Schenkungen und nach traditionellem Gewohnheitsrecht $\mathrm{zu}$ beurteilende bodenrechtliche Streitigkeiten zuständig sind (Art $110 \mathrm{JZOG).} \mathrm{Es} \mathrm{wird} \mathrm{hauptsächlich} \mathrm{durch} \mathrm{Einzelrichter} \mathrm{tätig} \mathrm{(Art} 24$ (3) S 1 JZOG). Wenn es um die Anwendung von traditionellem Gewohnheitsrecht geht, haben außerdem zwei aus der örtlichen traditionellen Führerschaft zu rekrutierende beisitzende Richter mitzuwirken (Art 24 (3) S 2 JZOG). Solche Gerichte sollen flächendeckend geschaffen werden (Art 24 (1) JZOG, allerdings stockte deren Einrichtung nicht nur aufgrund der Bürgerkriegshandlungen ${ }^{170}$. Berufungen gegen Entscheidungen der Friedensgerichte sind an die Landgerichte (Tribunaux de Grande Instance) zu richten (Art 114 JZOG), von welchen insgesamt 36 im Lande vorhanden sind. Außerdem gibt es zehn Appellationsgerichte. Auf allen Ebenen hat der Vertreter des Öffentlichen Interesses eine starke Stellung (Art 6 ff. JOZG). Ferner gibt es eine Militärgerichtsbarkeit ${ }^{171}$. Parallel zur staatlichen Gerichtsbarkeit existiert ein informelles Justizwesen ${ }^{172}$. Sicherheitsdienste, Milizen, lokale traditionelle Führer und moderne Warlords üben eine solche informelle Gerichtsbarkeit ebenso wie Bürgerkriegsparteien und andere Gruppierungen aus.

\section{b) Verfassungsrechtliche Regelung}

Zu den Innovationen der Verfassung von 2005 gehört die Schaffung einer Verwaltungsgerichtsbarkeit (Art $154 \mathrm{ff} \operatorname{Verf(2005))}$. Bis zu deren effektiven Einrichtung werden deren Kompetenzen jedoch vorläufig durch die ordentliche Gerichtsbarkeit mitausgeübt (Art 223, 224 Verf(2005)). Der bisher an der Spitze der ordentlichen Gerichtsbarkeit stehende Oberste Gerichtshof ${ }^{173}$ wurde durch die neue Verfassung in einen Kassationshof umgewan-

168

Langana et al.(Fn. 51), S. 7; zu den seinerzeitigen verfassungsrechtlichen Grundlagen vgl ua Art 40 Verf(1978).

Bha-Avira Mbiya, Conflits, juges et coutumes en Ituri, Kinshasa, 2004, S 3.

170 So waren 1993 erst an einem Viertel der vorgesehenen Standorte Friedensgerichte tätig, vgl Lamy, Le Code de la famille du Zaïre, Jahrbuch für afrikanisches Recht 7 (1993), S 45; International Commission of Jurists (Fn. 164), S. S 119.

172 Dibunda Kabuinji, Rôle et puvoir de la Cour Suprême de Justice au Zaïre, RevAnJurC 2 (1997), S $57 \mathrm{ff}$. 
delt (Art 149 (1), 157 ff., 223 Verf(2005)). Ferner wird eine Militärgerichtsbarkeit gebildet, welche für die Ahndung von Straftaten der Streitkräfte und der nationalen Polizei zuständig sein soll (Art 156 Verf(2005)). Das Staatssicherheitsgericht wurde abgeschafft (Art 225 (Verf(2005)). Der Oberste Richterrat setzt sich künftig ausschließlich aus Vertretern des Justizwesens zusammen (Art 152 Verf(2005); Vertreter der Politik (Art 147, 152, 153 Verf(2003)) sind nicht mehr beteiligt. Eine Umsetzung der Verfassungsbestimmungen in ein neues Justizgesetz befindet sich derzeit in Arbeit ${ }^{174}$.

\section{Justizgewährleistungen}

Angesichts des bisher vorherrschenden Rechtsvakuums geht die neue Verfassung detailliert auf wichtige Aspekte der Justizgewährleistung ein. Normiert werden u.a. die Grundsätze "nulla poene sine lege" (Art 17 (2) Verf(2005)), der Rückwirkung nur im Falle von Strafmilderungen (Art 17 (3) - (7) Verf(2005)), des Verbots der Sippenhaft (Art 17 (8) Verf(2005)), der Unschuldsvermutung (Art 17 (9) Verf(2005)) und des Anspruchs auf Rechtsbelehrung und Zugang zu Rechtsberatung (Art 18 (1) - (3) Verf(2005)). Im Falle eines Freiheitsentzugs von mehr als 48 Stunden ist ein Haftbefehl erforderlich (Art (Art 18 (4) Verf(2005)). Jedermann hat Anspruch auf den gesetzlichen Richter (Art 19 (1) Verf(2005)), auf ein Verfahren innerhalb angemessener Fristen (Art 19 (2) Verf(2005)) sowie auf Rechtsbeistand (Art 19 (3), (4) Verf(2005)). Gerichtsverfahren finden grundsätzlich öffentlich statt (Art 20 Verf(2005)); Urteile sind zu begründen (Art 21 (1) Verf(2005)) und Rechtsmitteln zugänglich (Art 21 (2) Verf(2005)). Die Klagemöglichkeit für Nichtregierungsorganisation gegen Menschenrechtsverletzungen, welche der Verfassungsentwurf des Senats vorgesehen hatte ${ }^{175}$, wurde in der endgültigen Fassung nicht berücksichtigt.

\section{Verfassungsgerichtsbarkeit}

a) Entwicklung

Der Oberste Gerichtshof versieht derzeit auch die Aufgaben eines Verfassungsgerichtshofs (Art 150 Verf(2003)). Dabei hat er mit seinen Entscheidungen in jüngster Zeit zunehmende Akzeptanz selbst in schwierigen Fragen wie der Verfassungsmäßigkeit des Vorstandes des Abgeordnetenhauses gefunden.

Medard Muyaya, Assemblée nationale: examen de proposition de loi organique portant statut des magistrates, Le Potentiel (Kinshasa) v 6.6.2005. 


\section{b) Verfassungsrechtliche Regelung}

Eine wichtige Rolle bei der Sicherung der Autorität der neuen Verfassung soll dem neu zu bildenden Verfassungsgerichtshof zukommen. Dieser soll aus 9 Mitgliedern bestehen, welche kongolesische Staatsangehörige sein und mindestens 15 Jahre juristische oder politische Erfahrung haben müssen (Art 158 ff Verf(2005)). Mindestens ein Drittel der Mitglieder müssen aus dem Justiz- und Anwaltswesen bzw der juristischen Hochschullehre hervorgegangen sein. Die Ernennung der Mitglieder erfolgt durch den Staatspräsidenten, wobei für jeweils ein Drittel der Mitglieder er selber, die beiden Parlamentskammern gemeinsam sowie der Oberste Richterrat das Vorschlagsrecht haben. Die Amtsdauer beträgt 9 Jahre, eine Wiederberufung ist unzulässig. Alle 3 Jahre findet eine Erneuerung zu jeweils einem Drittel statt, wobei das Los entscheidet, wer bei den ggf. auszuscheiden hat.

Zu seinen Kompetenzen gehören die Interpretation der Verfassung, die Prüfung der Verfassungsmäßigkeit von Gesetzen, Organstreitigkeiten zwischen Exekutiv- und Legislativgewalt auf zentraler oder Provinzebene, konkrete Normenkontrolle im Falle gerichtlicher Streitigkeiten sowie auf Verfassungsbeschwerde von Bürgern gegen Gesetze oder Verordnungen, die Wahlgerichtsbarkeit sowie die Durchführung von Strafverfahren gegen das Staatsoberhaupt oder den Ministerpräsidenten. Insbesondere konkrete Normenkontrolle und Verfassungsbeschwerde sind sehr viel weiter als in der bisherigen Verfassung (vgl Art 131, 150 ff.) ausgestaltet, so dass das Verfassungsgericht über weitreichende Möglichkeiten verfügen wird, die Gültigkeit von Gesetzen und Verordnungen aufzuheben oder einzuschränken $^{176}$. Bis zur Einrichtung des Verfassungsgerichts werden dessen Kompetenzen vom Kassationsgericht ausgeübt (Art 223 Verf(2005)).

(Der Beitrag wird im nächsten Heft fortgesetzt)

176 Kritisch dazu Kaossa/Kabamba/Lepautshi/Kasongo/Mvuezolo, Le Front Patriotique épingle les contradictions du Projet de Constitution, Le Phare (Kinshasa) v. 5.12.2006. 